\title{
Numerical Modeling of Active Flow Control in a Boundary Layer Ingesting Offset Inlet
}

\author{
Brian G. Allan*, Lewis R. Owens ${ }^{\dagger}$, and Bobby L. Berrier ${ }^{\ddagger}$ \\ NASA Langley Research Center, Hampton, VA, 23681, USA
}

\begin{abstract}
This investigation evaluates the numerical prediction of flow distortion and pressure recovery for a boundary layer ingesting offset inlet with active flow control devices. The numerical simulations are computed using a Reynolds averaged Navier-Stokes code developed at NASA. The numerical results are validated by comparison to experimental wind tunnel tests conducted at NASA Langley Research Center at both low and high Mach numbers. Baseline comparisons showed good agreement between numerical and experimental results. Numerical simulations for the inlet with passive and active flow control also showed good agreement at low Mach numbers where experimental data has already been acquired. Numerical simulations of the inlet at high Mach numbers with flow control jets showed an improvement of the flow distortion. Studies on the location of the jet actuators, for the high Mach number case, were conducted to provide guidance for the design of a future experimental wind tunnel test.
\end{abstract}

\section{Nomenclature}

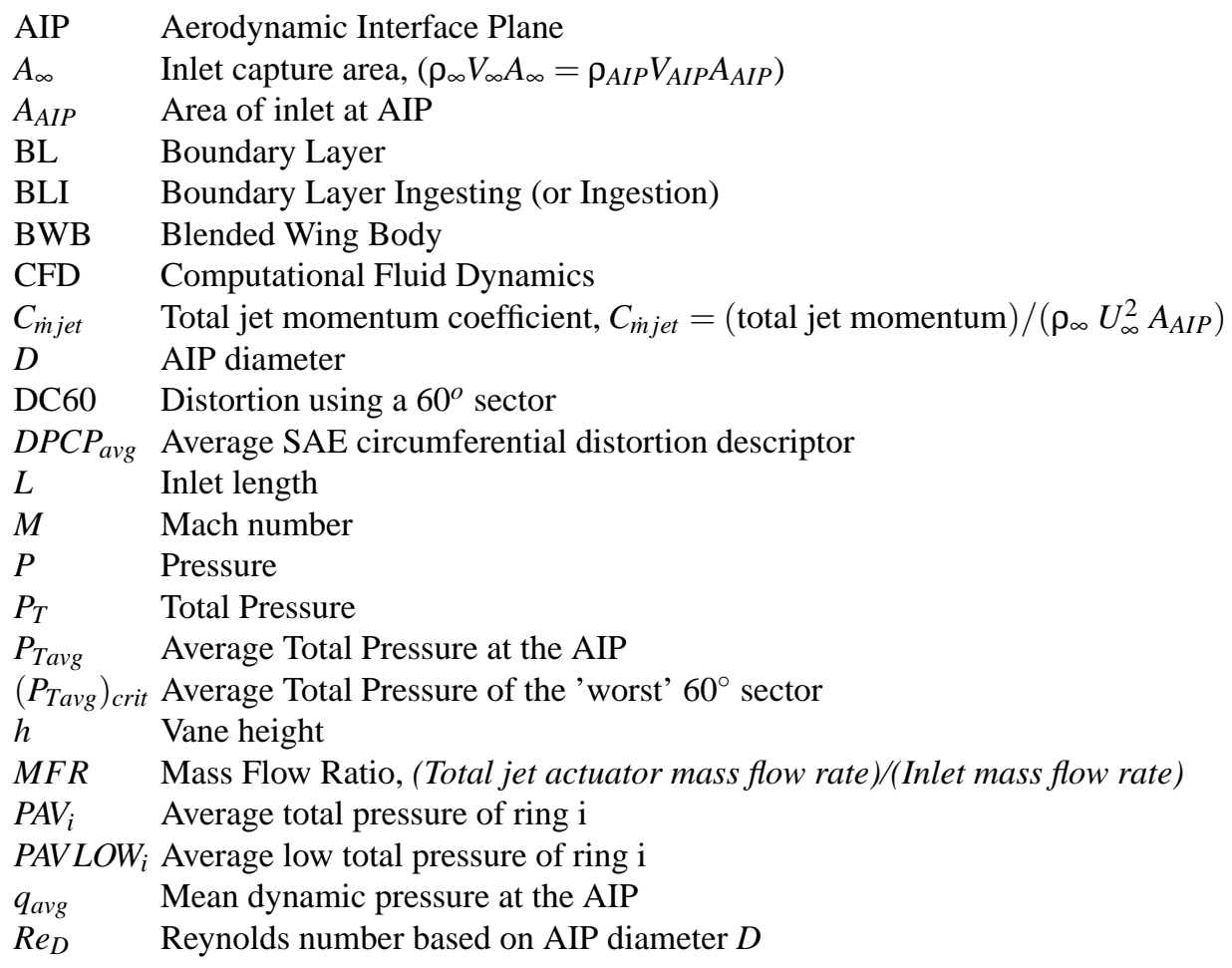

\footnotetext{
${ }^{*}$ Research Scientist, Flow Physics and Control Branch, NASA Langley Research Center, MS 170, Hampton, VA 23681, AIAA Senior Member. ${ }^{\dagger}$ Research Engineer, Flow Physics and Control Branch, NASA Langley Research Center, MS 170, Hampton, VA 23681, AIAA Senior Member. ${ }^{\ddagger}$ Associate Branch Head, Configuration Aerodynamics Branch, NASA Langley Research Center, MS 499, Hampton, VA 23681, and Fellow AIAA

This material is declared a work of the U.S. Government and is not subject to copyright protection in the United States.2004
} 


$\begin{array}{ll}s c f m & \text { Standard Cubic Feet per Minute } \\ U_{\infty} & \text { Free-stream Velocity, } \mathrm{m} / \mathrm{s} \\ u, v, w & \text { Velocity in } x, y, z \text { directions respectively, } \mathrm{m} / \mathrm{s} \\ \mathrm{VG} & \text { Vortex Generator } \\ x, y, z & \text { Cartesian axes } \\ \delta & \text { Boundary layer height } \\ \rho_{\infty} & \text { Free-stream Density }\end{array}$

\section{A. Subscripts}

$\begin{array}{ll}\text { avg } & \text { Average } \\ \infty & \text { Free-stream }\end{array}$

\section{Introduction}

$\mathbf{T}^{\mathrm{N}}$ an effort to reduce the environmental impact of commercial aircraft using revolutionary propulsion technologies, INASA initiated the Ultra Efficient Engine Technology (UEET) program. ${ }^{1}$ One of the elements of the UEET program is the application of flush mounted, boundary layer ingesting (BLI), offset inlets on the aft portion of an aircraft. System studies for the Blended Wing Body (BWB) transport have shown significant reductions in fuel burn by using this type of inlet. ${ }^{2}$ For the BWB vehicle, a BLI inlet placed on the upper rear surface of the wing would have a boundary layer to inlet height ratio of $30 \%$. The ingestion of such a large boundary layer coupled with the S-shaped offset of the inlet diffuser, results in a large flow distortion at the engine fan face. ${ }^{3,4,5}$ Experiments have shown that inlet distortion can be improved for the ingestion of a 30\% thick boundary layer to acceptable levels using flow control devices located inside the inlet. ${ }^{4}$

The current investigation studies the effects of vortex generating (VG) vanes and fluidic jets on inlet distortion and pressure recovery. The objectives of this investigation are twofold. The first objective is to validate the flow solver for the BLI offset inlet flow with and without flow control by fully modeling the VG vanes and jets. Once validated, simulations of the BLI inlet, with VG jets, will be performed for a future high Mach number experiment. The results from these simulations will provide guidance on the placement of jets in the BLI inlet. The second objective of this study is to provide additional insight into the flow physics of VG vanes and jets inside a BLI offset inlet. The insights gained from the simulations of the fully modeled control devices, will be used for the future validation of a source term actuator model. ${ }^{6}$ The source term model will significantly reduce the cost of simulating flow control devices in an inlet. Thus, making optimization studies for the placement and sizing of actuators in an inlet viable.

\section{Numerical Modeling}

The steady-state flow field for the BLI offset inlet with VG vanes and jets was computed using the flow solver code, OVERFLOW, ${ }^{7,8}$ developed at NASA. This code solves the compressible Reynolds averaged Navier-Stokes (RANS) equations using the diagonal scheme of Pulliam and Chaussee. ${ }^{9}$ The RANS equations are solved on structured grids using the overset grid framework of Steger et al. ${ }^{10}$ This overset grid framework allows for the use of structured grids for problems which have complex geometries. To improve the convergence of the steady-state solution, the OVERFLOW code also includes a low-Mach number preconditioning option and a multigrid acceleration routine, which were both used for the numerical simulations. All of the simulations in this study used Menter's two-equations (k- $\omega$ ) Shear-Stress Transport (SST) turbulence model. ${ }^{11}$ The SST turbulence model was found to be the best turbulence model option in OVERFLOW for the simulation of streamwise vortices embedded in a boundary layer. ${ }^{12}$

The numerical simulations were performed using the parallel

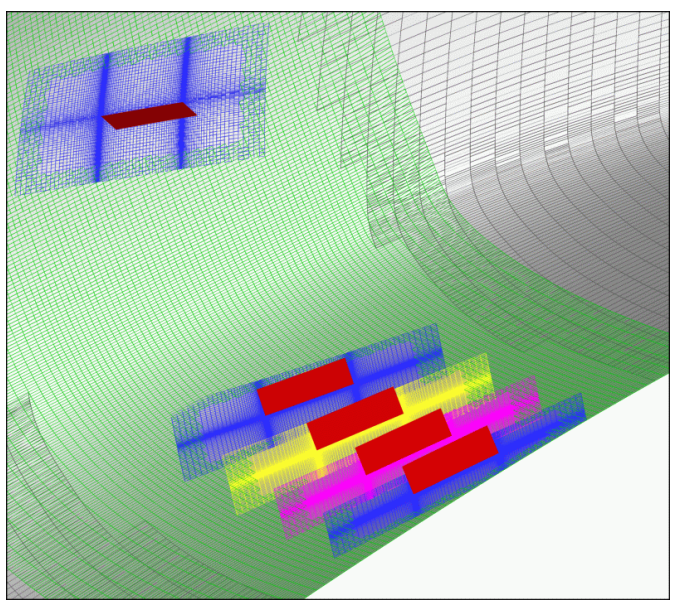

Figure 1. Overset grids for VG vanes inside the BLI inlet. 


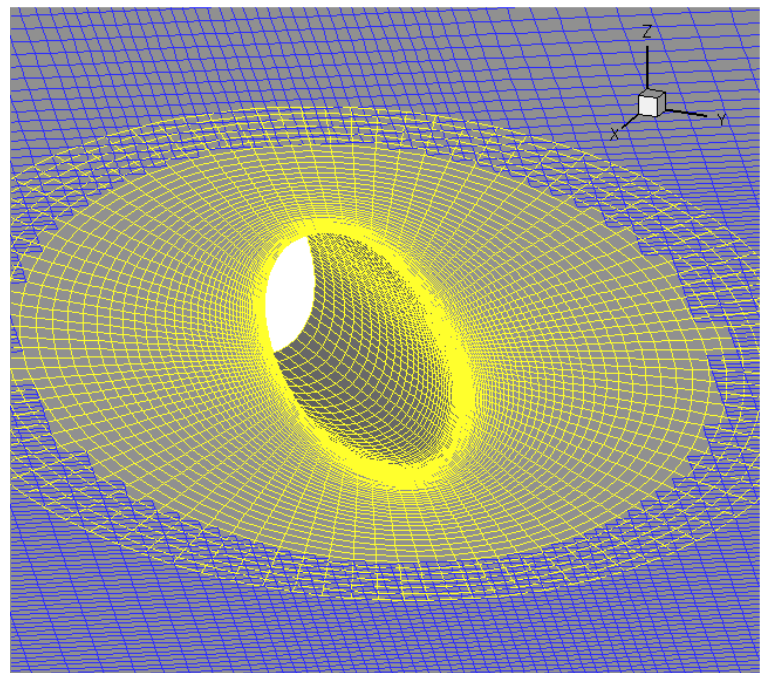

Figure 2. Close-up view of the overset nozzle grids for a VG jet inside of the BLI inlet.

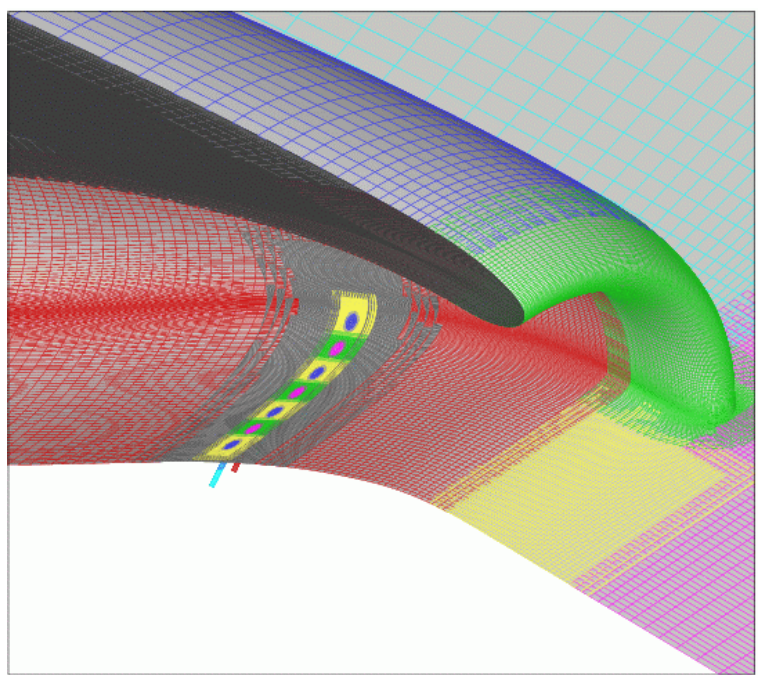

Figure 3. View of the overset nozzle grids and inlet grids.

version of the OVERFLOW code developed by Buning. ${ }^{13}$ This code uses the Message-Passing Interface (MPI) and can run on a tightly-coupled parallel machine or a network of workstations. The code distributes zones to individual processors and can split larger individual zones across multiple processors using a domain decomposition approach.

The structured overset grid system was generated using the Chimera Grid Tools package. ${ }^{14}$ Figure 1 shows a closeup view of the overset grids near the VG vanes on the inlet surface. The vanes were modeled as rectangular fins which was shown to be comparable to a fully modeled trapezoidal vane. ${ }^{12}$ Figure 2 shows a close-up view of the nozzle grid system for the $\mathrm{VG}$ jet simulation. The steady jet is skewed $90^{\circ}$ to the frees-tream flow and pitched at an inclined angle of $30^{\circ}$ to the surface. These pitch and skew angles for the jet result in the generation of a single streamwise vortex. This jet is simulated by modeling the nozzle plenum below the surface of the inlet. This simplifies the inflow boundary condition for the jet by letting the flow develop in the nozzle plenum and exiting at the duct surface. Figure 3 shows the inlet grids with the VG jet grids on the bottom surface of the inlet.

\section{Wind Tunnel Experiments}

The numerical simulations of the BLI offset inlet were compared to two experimental tests conducted at NASA Langley Research Center. ${ }^{3,4}$ One experiment was conducted in a low speed wind tunnel, the other in a high speed transonic tunnel. The low speed experiment evaluated both passive and active flow control devices; the high speed experiment investigated the baseline inlet at flight Mach and Reynolds numbers.

\section{A. Low Mach and Low Reynolds Number Experiment}

The low speed experiments for the BLI offset inlet were performed at NASA Langley's Basic Aerodynamics Research Tunnel (BART). ${ }^{4}$ This wind tunnel had a free-stream Mach number of 0.15 as was able to generate a boundary layer to inlet height ratio of $36 \%$. The experimental test had a Reynolds number of $\operatorname{Re}_{D}=0.54 \cdot 10^{6}$, where $D=6.0$ inches and is equal to the aerodynamic interface plane (AIP) diameter. This experiment showed the improvement of flow distortion using VG vanes and jets located inside the inlet. The data from this experiment will be used to compare and validate the flow solver for low Mach number flows. Further information on the experiment is provided by Gorton et al. $^{4}$

\section{B. High Mach and High Reynolds Number Experiment}

The high Mach and Reynolds number experiments were conducted at NASA Langley's 0.3-Meter Transonic Cryogenic Tunnel (0.3-Meter Tunnel) for the BLI offset inlet. ${ }^{3}$ Experimental data was obtained for the baseline inlet case for a 
free-stream Mach number range from 0.25 to 0.83 . The experimental test had a Reynolds number range of $R e_{D}=6.8$. $10^{6}$ to $14.3 \cdot 10^{6}$, where the AIP diameter, $D=2.448$ inches. This experiment was able to test the BLI inlet at actual flight Mach and Reynolds numbers as expected for the BWB aircraft application. This experiment generated a boundary layer of approximately $30 \%$ of the inlet height ratio.

\section{BLI Offset Inlet Geometry}

The BLI offset inlet was designed by Boeing under contract to NASA in order to provide an inlet that would be representative of designs considered for the commercial version of the BWB aircraft. The inlet geometry is of a generic nature and is an open geometry that can be used for computational validation and experimental investigations. Figure 4 shows the side view of the S-shaped inlet for the BART experiment. The inlet for the BART experiment is $6.129 \%$ of full scale and the inlet model for the 0.3-Meter Tunnel experiment was built to $2.5 \%$ scale. The inlet cross section transitions from a Dshape at the entrance to circular shape at the AIP. Figure 4 also shows the location of the AIP where the total pressure rake was located and where the engine face would be when attached to the inlet. This figure also shows the location of the VG plane

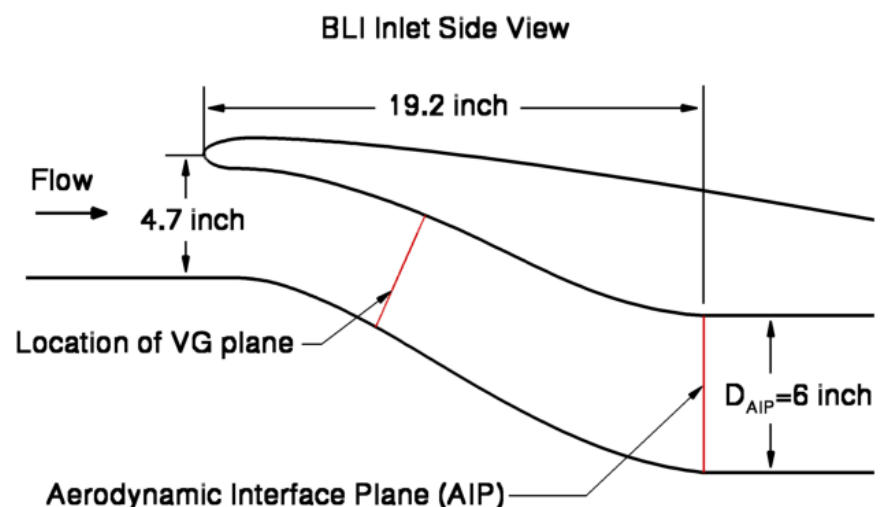
where the passive and active flow control devices were placed for the BART experiment. This location has 32 jet orifices placed circumferentially on the inlet surface where different combinations of jet locations were evaluated by Gorton et al. ${ }^{4}$

\section{Distortion Descriptors}

Two different distortion descriptors were used to evaluate inlet performance. The first inlet performance parameter is the average SAE circumferential distortion descriptor defined in the Aerospace Recommended Practice (ARP) 1420 standard. In this paper the average distortion intensity is given by $D P C P_{\text {avg }}$ and defined in (1).

$$
D_{P C P_{\text {avg }}}=\frac{1}{N_{\text {rings }}} \sum_{i=1}^{N_{\text {rings }}} \text { Intensity }_{i}
$$

where $i$ is the ring number on the AIP rake and $N_{\text {rings }}$, the total number of rings. The Intensity for each ring is defined in (2).

$$
\text { Intensity }_{i}=\frac{P A V_{i}-P A V L O W_{i}}{P A V_{i}}
$$

where $P A V_{i}$ is the average total pressure of ring $i$ and $P A V L O W_{i}$, the average of the low total pressure region below $P A V_{i}$.

The second engine face distortion descriptor used is DC60 defined in (3).

$$
D C 60=\frac{P_{\text {Tavg }}-\left(P_{\text {Tavg }}\right)_{\text {crit }}}{q_{\text {avg }}}
$$

The $D C 60$ distortion descriptor is computed from the mean total pressure at the AIP, $P_{\text {Tavg }}$, the mean dynamic pressure, $q_{\text {avg }}$, and the mean total pressure in the 'worst' $60^{\circ}$ sector, $\left(P_{\text {Tavg }}\right)_{\text {crit. }}{ }^{15}$ Where the 'worst' $60^{\circ}$ sector is the sector with the lowest mean total pressure. Unlike the $D P C P_{\text {avg }}$ distortion descriptor, $D C 60$ is scaled by the average dynamic pressure at the AIP. For experimental data, $q_{\text {avg }}$ is typically estimated using static pressure measurements on the surface of the inlet around the rake. The mean of all the static pressure measurements is then used to estimate the Mach number at the total pressure rake locations which is then used to compute $q_{\text {avg. }}$. For the high Mach number cases with BLI, it was discovered using data from the numerical simulations, that this approach to estimating $q_{\text {avg }}$ resulted in a 7 to 


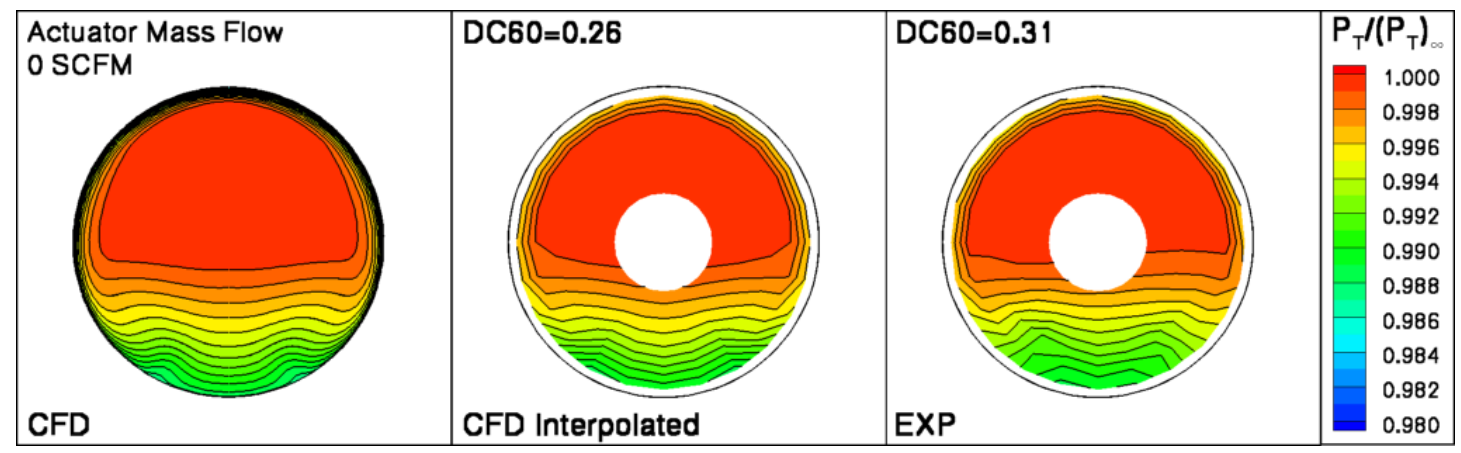

Figure 5. A comparison of the numerical and experimental results for the BLI inlet for the baseline low speed case, $M_{\infty}=0.15$, with a duct flow rate of $1800 \mathrm{scfm}$. These contour plots show the total pressure ratio at the AIP where the CFD results are interpolated onto the 120 probe locations used in the experiment.

$14 \%$ error. This estimation error in $q_{a v g}$ is then carried over to the $D C 60$ distortion values. For the low Mach number experiments, the estimation error for $q_{\text {avg }}$ was 1 to $4 \%$ since the static pressure did not vary as much across the AIP as in the high Mach number case. All of the DC60 results for the numerical simulations and experimental data followed the practice of computing $D C 60$, where $q_{\text {avg }}$ is estimated from a mean static wall pressure and the total pressures at the rake locations. The static and total pressures used to compute the distortion descriptors for the numerical simulations were also interpolated at same locations as the experimental data. This way the numerical results can be evaluated at the same resolutions as the experimental data.

\section{Results and Discussions}

\section{A. Baseline: Low Mach and Low Reynolds Number Case}

The baseline case for the low Mach and Reynolds number experiment had a free-stream Mach number of 0.15 with a Reynolds number of $R e_{D}=0.54 \cdot 10^{6}$. The duct mass flow rate was held fixed at $1800 \mathrm{scfm}$ for all low Mach number experimental data presented in this paper. Figure 5 shows a comparison between the numerical and experimental results for the low Mach and Reynolds number baseline case. This figure shows the contour plots of the total pressure ratio, $P_{T} /\left(P_{T}\right)_{\infty}$, at the AIP. The experimental data was taken using a 40 probe total pressure rake located at the AIP. This rake was then rotated by 15 and 30 degrees resulting in 120 total pressure measurements. The high resolution of the numerical results in Fig. 5 are interpolated to the same resolution as the experimental data. The 120 total pressure measurements are then used to compute a distortion value using the DC60 method. ${ }^{15}$ The experiment had a DC60 value of 0.30 where the numerical simulation was predicting 0.26 . This comparison shows a difference of $13 \%$ for the low speed baseline results. Overall the contour plots compare very well for the baseline case showing similar flow distortion patterns.

\section{B. Baseline: Low Mach and High Reynolds Number Case}

Numerical simulations were compared to the high speed experiments taken in the 0.3-Meter Transonic Cryogenic Tunnel at two free-stream Mach numbers. The lower free-stream Mach cases are shown in Fig. 6 where $M_{\infty}=0.25$ with mass flow rates of 6.38 and $7.10 \mathrm{lbm} / \mathrm{s}$. These duct flow rates result in an acceleration of the flow inside the duct for an average Mach of 0.35 at the AIP for the low mass flow rate and 0.39 for the high mass flow rate case. These two cases also have Reynolds numbers, based on the AIP diameter, of about $\operatorname{Re}_{D}=6.9 \cdot 10^{6}$.

Since the BLI inlet was modeled on a flat plate, the effects of the tunnel walls were not accounted for in the numerical simulations. In order to match the experimental flow conditions, the flat plate length ahead of the inlet and the free-stream Mach number were adjusted, matching the boundary layer velocity measured near of the inlet face. The BL rake was located 3.784 inches from the centerline of the inlet and 0.10 inches upstream of the cowl highlight. The rake was approximately 1.67 inches away from the cowl outer surface and had a height of 0.58 inches. In the first numerical simulation the flat plate length was determined to match the experimental BL height. This simulation used 


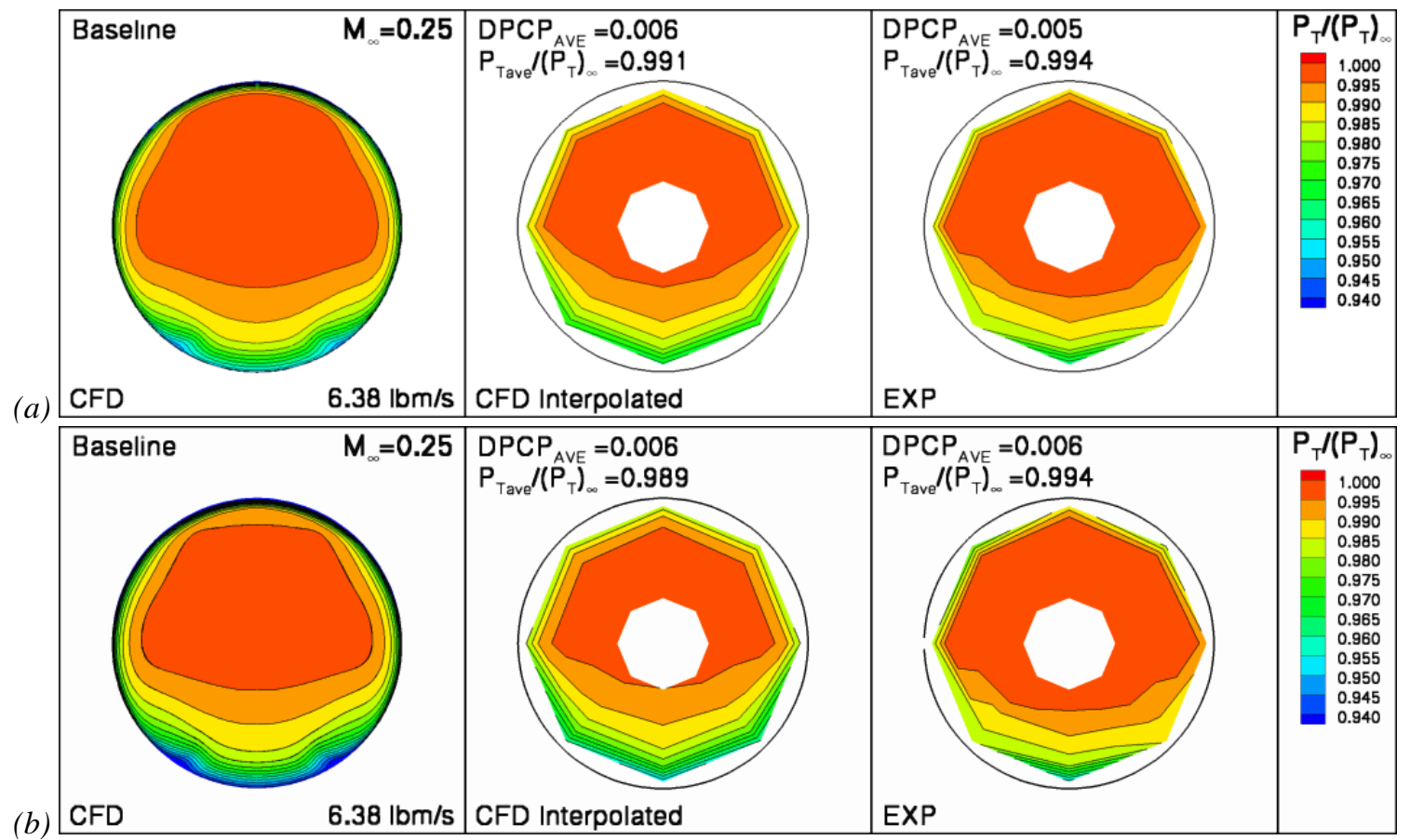

Figure 6. Comparison of numerical and experimental results for the BLI inlet for the baseline flow taken in the 0.3-Meter Transonic Cryogenic Tunnel. Case (a) has a free-stream Mach of 0.25 with a $\operatorname{Re}_{\mathrm{D}}=6.8 \cdot 10^{6}$ and a duct mass flow rate of $6.38 \mathrm{lbm} / \mathrm{s}$. Case $(b)$ has a free-stream Mach of 0.25 with a $\operatorname{Re}_{D}=6.9 \cdot 10^{6}$ and a duct mass flow rate of $7.10 \mathrm{lbm} / \mathrm{s}$.

the free-stream Mach number given by the experiment which was measured upstream of the test section. Figure 7 shows the BL rake data for the high and low Mach number cases at a given inlet mass flow rate. These figures show how the Mach number at the BL edge for the numerical simulations was slightly higher than the experiment. The free-stream Mach number for the simulations was then adjusted to match the velocity measured at the BL rake. The free-stream Mach number for the $M_{\infty}=0.25$ case was adjusted to $M_{\infty}=0.234$ in the numerical simulation, producing a better match to the BL velocity as shown in Fig. 7 . In the $M_{\infty}=0.833$ case, the free-stream Mach number was reduced to $M_{\infty}=0.784$ which resulted in a better match to the BL velocity.

The BL comparison in Fig. 7 shows how the BL profile is slightly different in the experiment for the high Mach number case as compared to the numerical simulations. The BL in the experiment has less energy near the wall than the numerical simulation. The BL for the experiment was generated from the tunnel wall and not from a splitter plate, which may account for the difference in the BL profiles.

The flow distortion for the high Reynolds number experiments was calculated using the $D P C P_{\text {avg }}$ descriptor. The flow distortion for the lower mass flow rate case had a $D P C P_{a v g}=0.005$ as compared to 0.006 predicted by the numerical simulation. The numerical simulation had a slightly lower pressure recovery of $P_{\text {Tavg }} /\left(P_{T}\right)_{\infty}=0.991$ which compares well to the experimental value of 0.994 . The distortion for the higher mass flow rate was identical for the simulation and the experiment having at value of $D P C P_{\text {avg }}=0.006$. As in the lower mass flow rate case, the numerical results showed a slightly lower pressure recovery of 0.989 as compared to the experiment which measured 0.994 . Figure 6 shows a comparison of the total pressure ratio contours which compare very well between the experiment and numerical results.

\section{Baseline: High Mach and High Reynolds Number Case}

The high free-stream Mach number cases are shown in Fig. 8 where $M_{\infty}=0.833$ and the duct mass flow rates were 5.0 and $6.0 \mathrm{lbm} / \mathrm{s}$. The low mass flow case had a $\operatorname{Re}_{D}=14.3 \cdot 10^{6}$ and the high mass flow rate case a $\operatorname{Re}_{D}=13.8 \cdot 10^{6}$. The comparison of distortion patterns for the high free-stream Mach number cases are good overall as seen in Fig. 8 . For the low mass flow rate case in Fig. $8 a$, a $D P C P_{\text {avg }}$ of 0.040 was predicted by the numerical simulations as compared to 0.034 measured in the experiment. The numerical simulations predict a distortion about $18 \%$ larger than was seen 

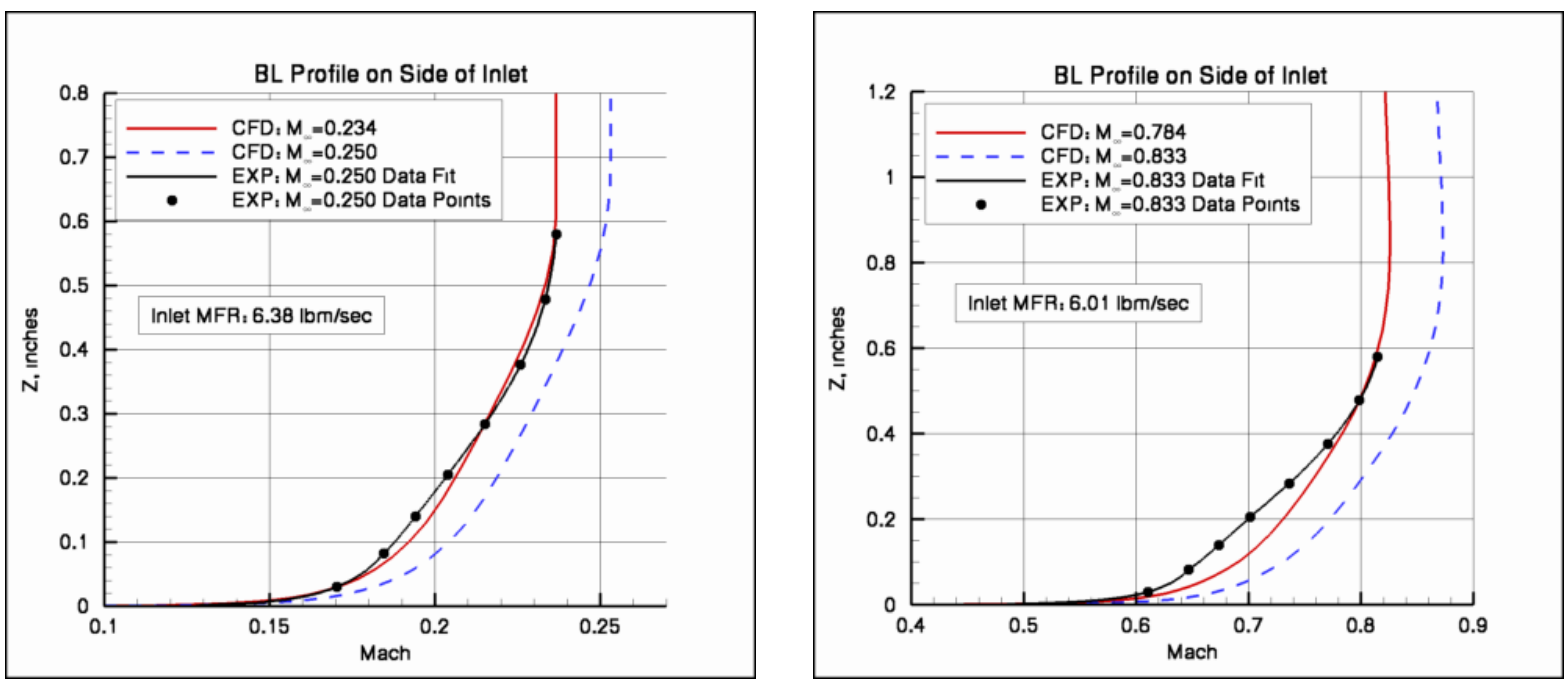

Figure 7. A comparison of the boundary layer profile on the side of the inlet for the experiment and the numerical simulation. The rake is located 0.10 inches upstream of the highlight on the inlet cowl, 3.784 inches from the inlet centerline and approximately 1.67 inches from the outer surface of the inlet cowl. These figures show how the free-stream Mach number for the simulation needed to be adjusted to match the velocity profile at the BL rake.

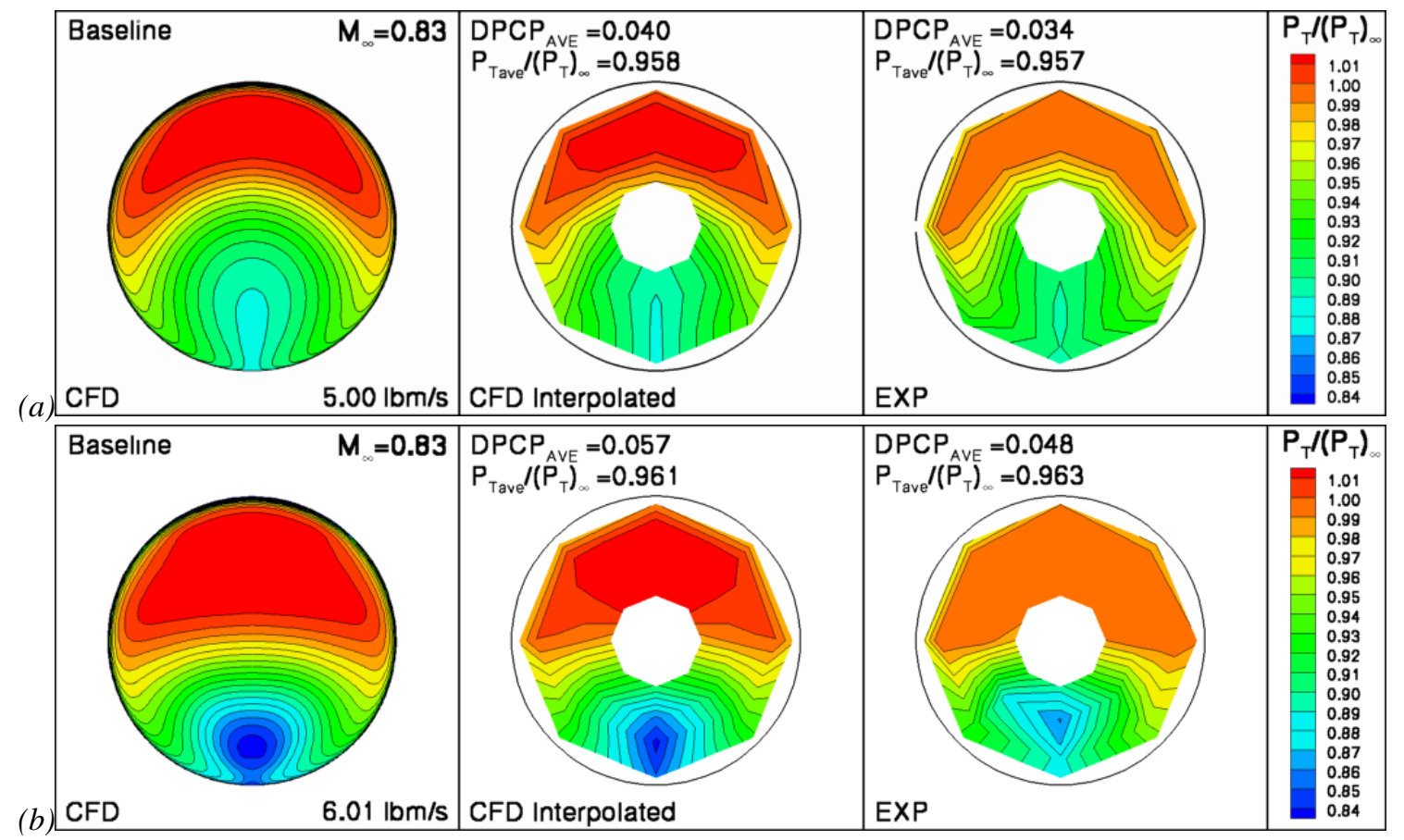

Figure 8. Comparison of numerical and experimental results for the BLI inlet for the baseline flow taken in the 0.3-Meter Transonic Cryogenic Tunnel. Case (a) has a free-stream Mach of 0.833 with a $R_{\mathrm{D}}=14.3 \cdot 10^{6}$ and a duct mass flow rate of $5.00 \mathrm{lbm} / \mathrm{s}$. Case $(b)$ has a free-stream Mach of 0.833 with a $\operatorname{Re}_{\mathrm{D}}=13.8 \cdot 10^{6}$ and a duct mass flow rate of $6.01 \mathrm{lbm} / \mathrm{s}$. 


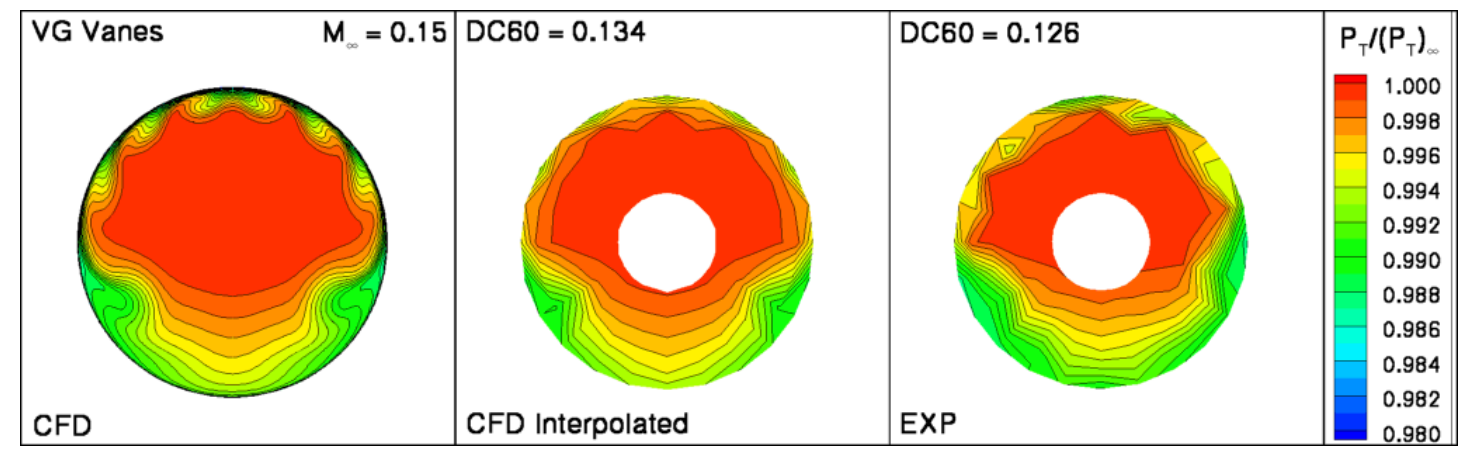

Figure 9. A comparison of the numerical and experimental results for the BLI inlet using VG vanes for the low speed case. These contour plots show the total pressure ratio at the AIP. The CFD results were interpolated onto the 120 probe locations used in the experiment.

in the experiment. This higher distortion value can be explained by looking at the total pressure contour plots in Fig. $8 a$. Comparing the two plots it can be seen that the numerical simulation has a higher peak total pressure and a lower minimum. This larger difference between the maximum and minimum total pressure ratios, for the numerical solution, produces a larger distortion value.

The higher mass flow rate case shown in Fig. $8 b$ shows the same trend in the distortion value where $D P C P_{\text {avg }}=$ 0.057 for the numerical simulation and 0.048 for the experiment. The difference in the low and high total pressure values between the CFD and experiment can also be seen by comparing these contour plots. Despite the difference in the distortion values, the total pressure recovery between the experiment and numerical solutions compare well. The low mass flow rate case in Fig. $8 a$ shows a $P_{\text {Tavg }} /\left(P_{T}\right)_{\infty}=0.958$ from CFD results as compared to the experimental value of 0.957 . The high mass flow rate case in Fig. $8 b$ shows a $P_{\text {Tavg }} /\left(P_{T}\right)_{\infty}=0.961$ for the numerical simulation and 0.963 for the experiment.

\section{Controlled: Low Mach and Low Reynolds Number Case}

The BART experiment evaluated both passive and active flow control devices. A numerical simulation of one passive control device configuration was compared to the experiment as well as a single active flow control configuration. In the active flow control configuration, several numerical simulations were compared to varying actuator mass flow rates.

\section{Passive Control Devices: VG Vanes}

While the focus of the BART experiments was active flow controls, a configuration using passive flow control devices was tested for performance comparisons and for validation of flow solver codes. The vanes were located on the same plane as the VG jets with eight vanes located at the bottom of the duct and six on the upper part of duct. The vanes had a tapered leading edge with a height of 3/16 inches and a length of 3/4 inches and were placed at an angle of $20 \mathrm{deg}$ with respect to the center line of the duct. The vanes were located along the VG plane shown in Fig. 4 and angled in such a way as to induce the flow away from the bottom of the duct centerline toward the top. The vanes used in the numerical simulation were rectangular with the same height and area. It was discovered by Allan ${ }^{12}$ that when modeling trapezoidal vanes submerged in a boundary layer with rectangular vanes, that one should use the same height as well as the same area. A comparison of the numerical simulation of the BLI inlet with VG vanes and the experiment is shown in Fig. 9. This figure shows the total pressure ratio contours at the AIP. The numerical solution clearly shows the low total pressure regions from the streamwise vortices created by the upper VG vanes. There is also a region on the lower side of the AIP where the streamwise vortices from the bottom VG vanes and the boundary layer merge. These regions can be related to the experimental contour plots as well as the interpolated CFD data. The numerical results predict a flow distortion of $D C 60=0.134$ and compare very well to the experimental value of 0.126 . This comparison shows how the numerical solution can be used to gain a better insight into the flow features generated by the flow control devices. 
Numerical simulations for the active flow control devices are compared to the best jet configuration found in the experiments of Gorton et al. ${ }^{4}$ The best VG jet configuration was found by trying different combinations of the 32 possible jet locations. The best configuration was defined as the configuration which was able to obtain a desired DC60 value of 0.10 using the lowest possible mass flow rate. It was found by Gorton et al. ${ }^{4}$ that the best configuration was obtained by using fourteen jets on the bottom of the inlet. The VG jets were aligned $90^{\circ}$ to the flow and inclined $30^{\circ}$ to the surface of the duct with a orifice diameter of 0.060 inches. The numerical solution for the VG jet case was modeled using 29 grids resulting in a total of 11 million grid points.

The experimental results presented here are for pulsed jets at $50 \mathrm{~Hz}$ with an $80 \%$ duty cycle; the numerical simulations assume steady blowing jets. From the experiments, it was found that there was less than $1 \%$ difference in the distortion values between steady blowing jets and the pulsed jets

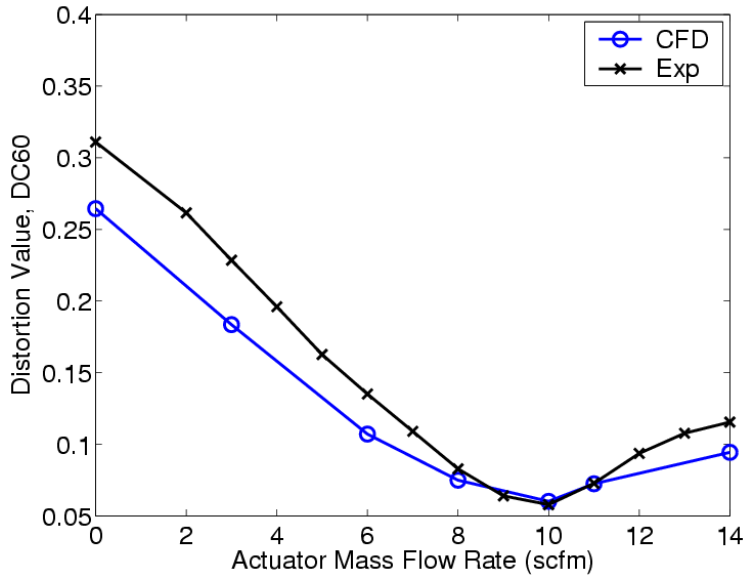

Figure 10. A comparison of the DC60 distortion values between the numerical and experimental results for the low free-stream Mach number BLI inlet case with VG jets.

at $50 \mathrm{~Hz}$ with an $80 \%$ duty cycle at the same mass flow rate. Therefore the numerical steady blowing jet results are compared to the experimental pulsed jet data with the assumption that the effects from the pulsed jets are equivalent to a steady blowing jet at the same mass flow rate.

A mass flow sweep was performed for the $\mathrm{VG}$ jets at $M_{\infty}=0.15$ and for a constant duct mass flow rate of $1800 \mathrm{scfm}$. Figure 10 shows the comparison of the DC60 distortion values between the experiment and the numerical solutions. This comparison shows that the flow solver was able to capture the correct trend and was able to predict the point where DC60 starts to climb with increasing mass flow rate. It can also be seen that the numerical simulations under predicted the distortion values at lower actuator flow rates by an average of $13 \%$.

The surface $C_{P}$ measured on the duct's upper and lower centerline is shown in Figs. 11 and 12 for the controlled inlet case. Figure 11 compares the surface $C_{P}$ for the low actuator mass flow rate case of $3 \mathrm{scfm}$ where the inlet leading edge is located at $x=0$ and the actuators at $x=6.6$ inches. This plot shows that the numerical solution for the surface pressure matched the experiment with a slight offset. Figure 12 shows the surface $C_{P}$ for a high actuator mass flow rate of 14 sc fm. The CFD surface pressure distribution compares well with the experimental data with the numerical simulation capturing the low pressure region near the jets.

The total pressure ratio contours for the actuator mass flow sweep are shown in Fig. 13. Overall, the contour plots for the interpolated CFD and the experiment compare very well. The location and size of the low pressure regions

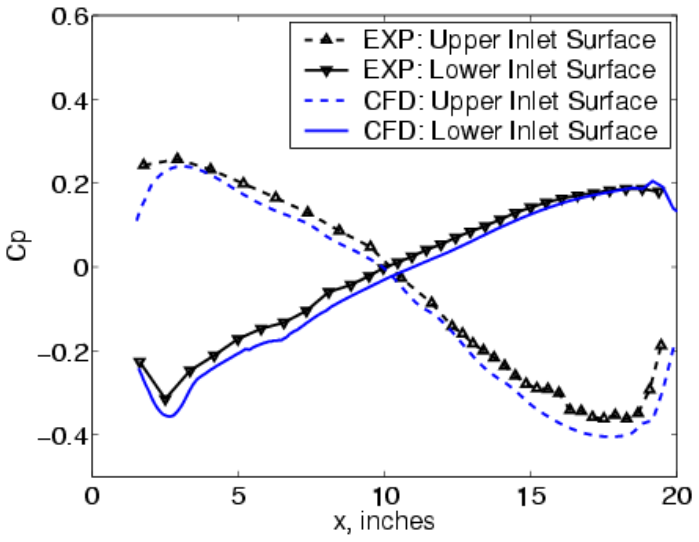

Figure 11. Upper and lower duct centerline surface $C_{P}$ comparison for the controlled BLI inlet with flow control jets. The data is from the BART experiment with a low actuator mass flow rate of $3 \mathrm{scfm}, M_{\infty}=0.15$ and a duct mass flow rate of $1800 \mathrm{scfm}$.

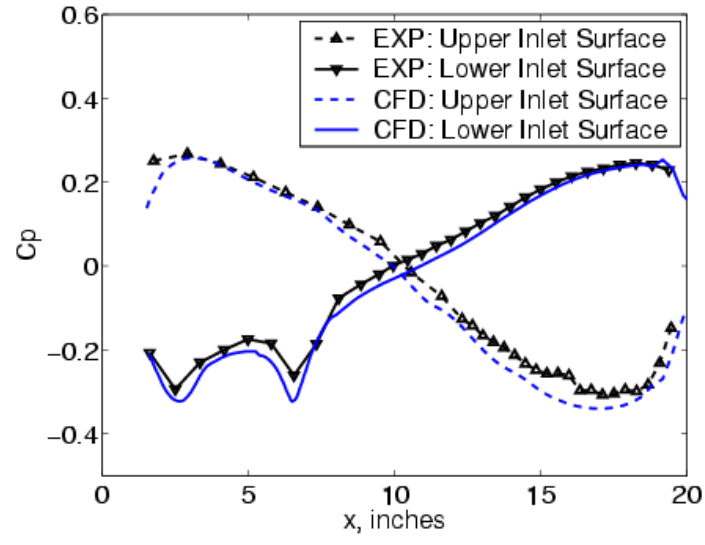

Figure 12. Upper and lower duct centerline surface $C_{P}$ comparison for the controlled BLI inlet with flow control jets. The data is from the BART experiment with a high actuator mass flow rate of $14 \mathrm{scfm}, M_{\infty}=0.15$ and a duct mass flow rate of $1800 \mathrm{scfm}$. 


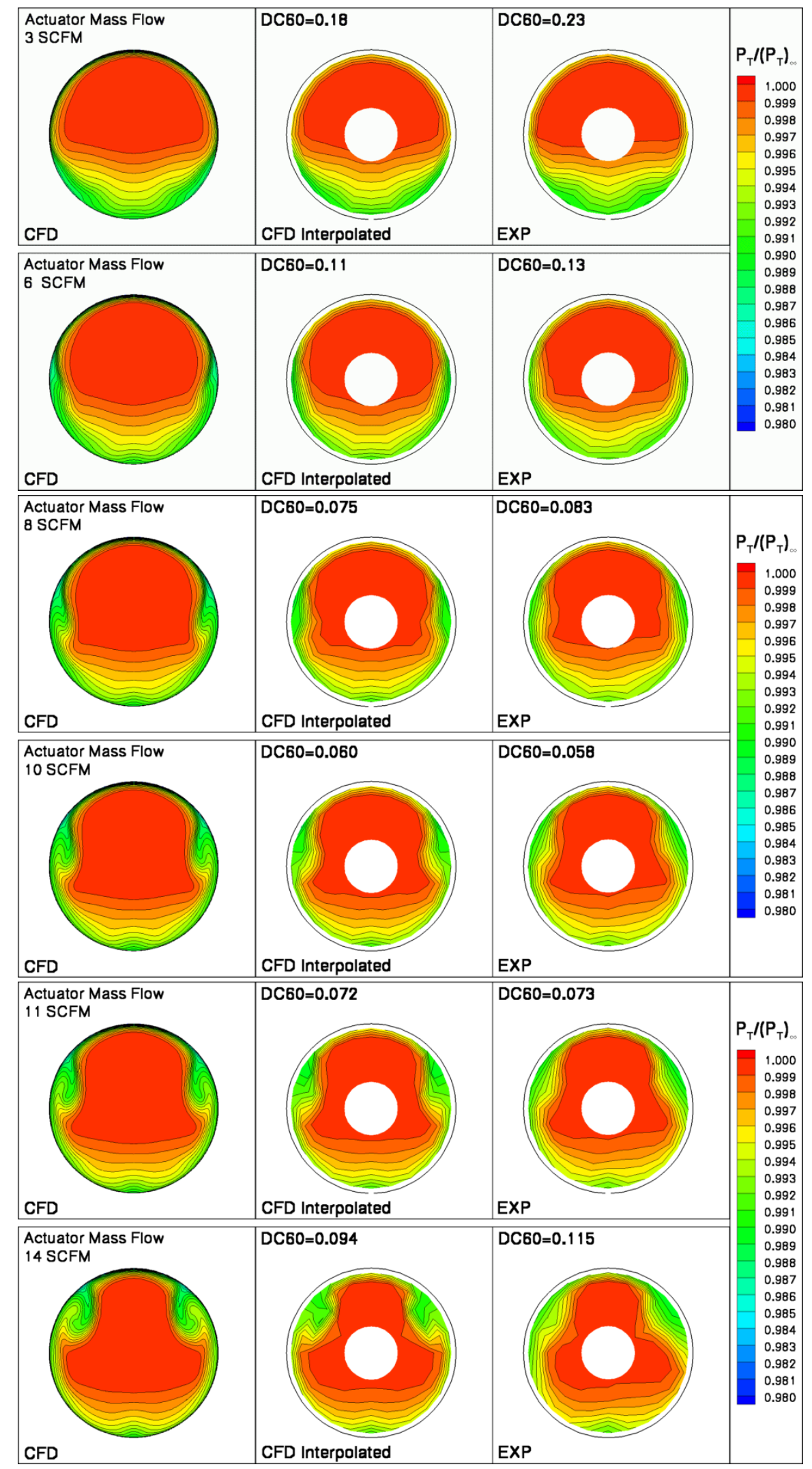

Figure 13. A comparison of the numerical and experimental results for the BLI offset inlet, using VG jets at various mass flows, for the low Mach number case. These contour plots show the total pressure ratio at the AIP. The CFD results were interpolated onto the 120 probe locations used in the experiment. 


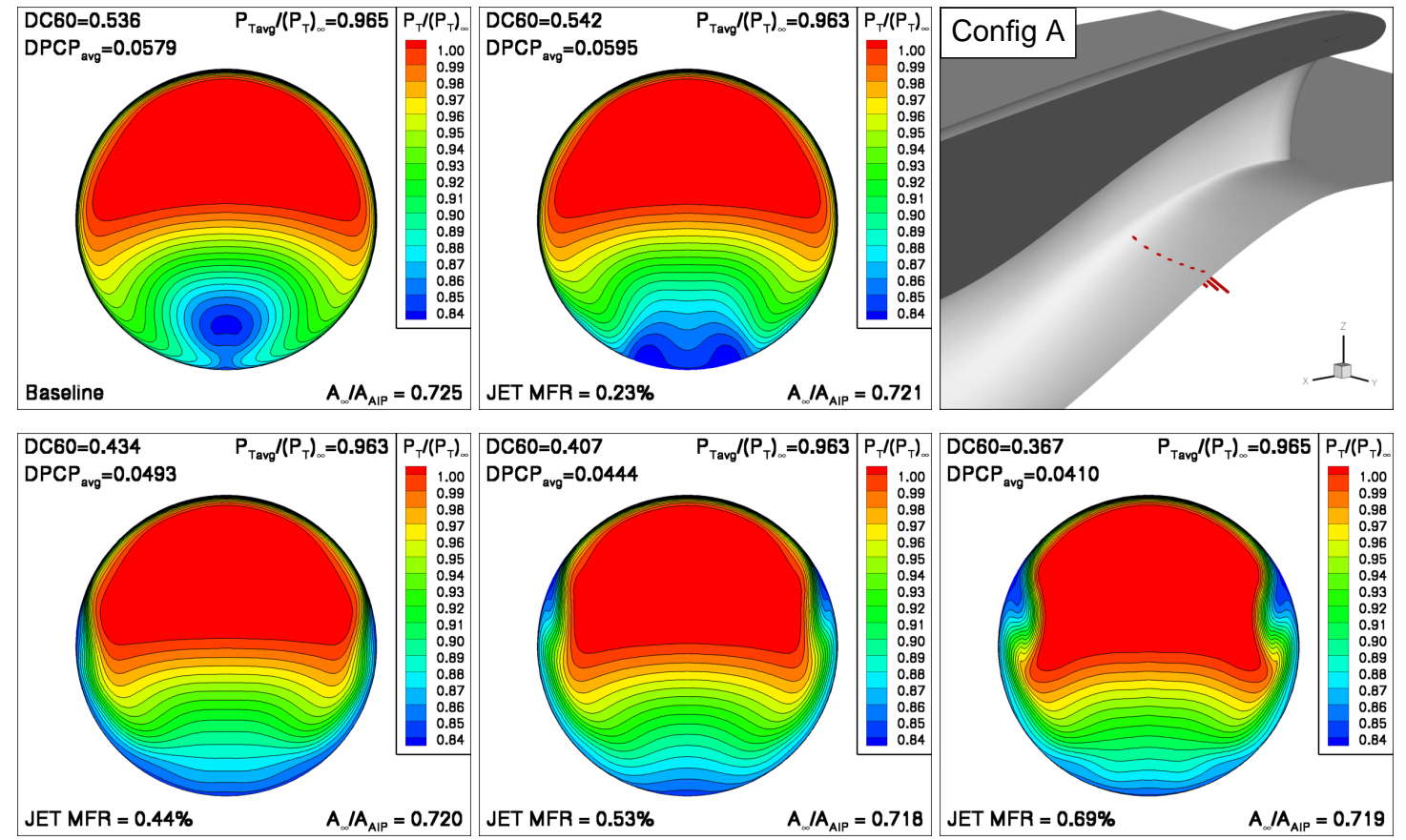

Figure 14. Total pressure ratio contours of the numerical simulations for the BLI inlet with $\mathbf{1 4}$ control jets for varying jet mass flow ratios where $M_{\infty}=0.784$ and $R_{D}=13.8$ million. The location of the control jets are shown and are at the same location as the jets in the low Mach case where they are on the bottom of the duct at $x / L=0.34$.

on the sides of the AIP match up well with the experiment. The shape of this region does differ slightly for the low actuator mass flow rates. As the mass flow is increased, the shape of this low pressure region starts to differ more between CFD and experiment. The difference in the size and shape of this region is most likely related to the size of a streamwise vortex as seen in the high resolution CFD results. Finally, the total pressure contours at the bottom of the AIP compare very well with the experiment for each of the mass flow rates.

\section{E. Controlled: High Mach and High Reynolds Number Case}

The performance of the control jets were evaluated for the high Mach and Reynolds number case using the same CFD approach as in the low Mach number case. These simulations will provide guidance on the placement of the control jets for a future high Mach number BLI inlet experiment. As in the low Mach case the jets are aligned $90^{\circ}$ to the flow and inclined $30^{\circ}$ to the surface of the duct with a orifice diameter of 0.040 inches. These simulations are for the 2.5\% scale BLI inlet geometry used in the 0.3-Meter Transonic Cryogenic Tunnel test. The performance of the jets are evaluated for the $M_{\infty}=0.833$ case in Fig. $8(b)$. Of all of the flow conditions considered, this case had the largest $D P C P_{\text {avg }}$ distortion value. In all of the high Mach number controlled simulations the back pressure for the inlet is fixed at baseline value. Therefore, there was no attempt to adjust the exit pressure in order to maintain a constant inlet mass flow rate for varying jet mass flows, as was done in the low Mach and low Reynolds number cases.

Several actuator configurations were evaluated for the high Mach number case and will be referred to as configurations A through G. Jet configuration A, shown in Fig. 14, was the first to be evaluated and is the same jet layout used in the low Mach number cases. The actuator placement for configuration A consisted of a single row of jets at $x / L=0.34$, where there are 7 jets on each side inlet centerline. These jets are aligned such that they are blowing from the centerline outwards. The simulation of the jets was performed at four different mass flow rates. The total actuator mass flow rates are given in terms of a jet mass flow ratio (MFR) which is defined in (4).

$$
\text { Jet MFR }=\frac{\text { Total Jet Mass Flow Rate }}{\text { Inlet Mass Flow Rate }}
$$

The highest jet MFR for configuration A was $0.69 \%$ and demonstrated a reduction of the baseline $D P C P_{\text {avg }}$ distortion 


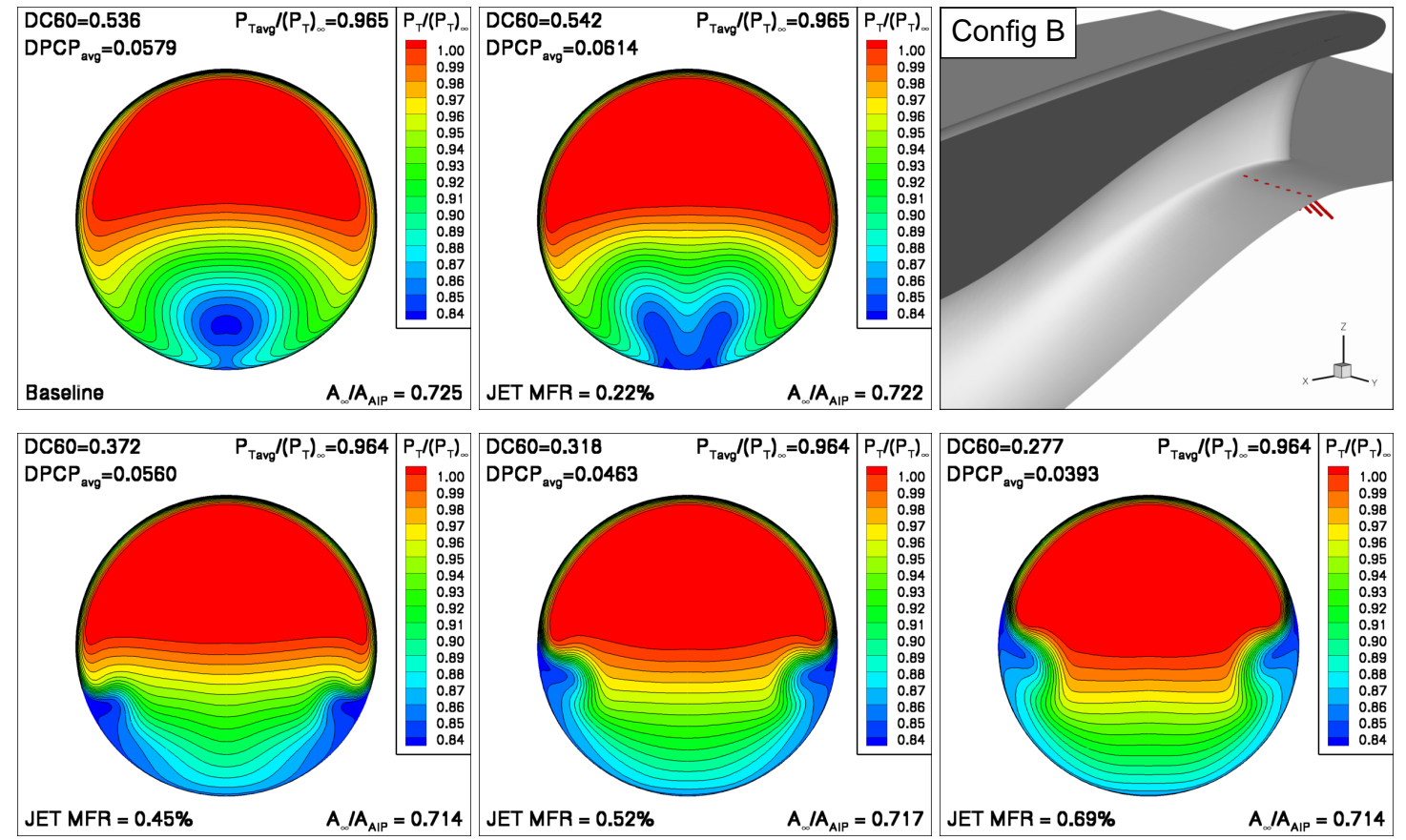

Figure 15. Total pressure ratio contours of the numerical simulations for the BLI inlet with $\mathbf{1 4}$ control jets for varying jet mass flow ratios where $M_{\infty}=0.784$ and $\operatorname{Re}_{D}=13.8$ million. The jets are placed near the entrance of the inlet at $x / L=0.13$.

value from 0.0579 to 0.0410 . The $D C 60$ distortion descriptor was reduced from 0.536 to 0.367 . The pressure recovery, $P_{\text {Tavg }} /\left(P_{T}\right)_{\infty}$, was not effected by the control jets and remained at about 0.965 . The low jet MFR $=0.23 \%$ shows a spreading out of the concentrated low pressure region seen at the bottom of the AIP in the baseline case. The intermediate jet $\mathrm{MFR}=0.44 \%$ case shows the low pressure region smeared out along the bottom of the AIP with very little thinning of the ingested BL. The $0.52 \%$ jet MFR case starts showing a pooling of a low pressure region on the sides of the AIP and a slight thinning of the low pressure region. The highest MFR case of $0.69 \%$ shows larger low pressure regions on the side of the inlet AIP with a thinning of the low pressure region on the bottom of the inlet.

Figure 14 also shows the ratio of the capture area, $A_{\infty}$, to the inlet AIP area, $A_{A I P}$, where $A_{\infty}$ is defined by (5).

$$
A_{\infty} \rho_{\infty} V_{\infty}=A_{A I P} \rho_{A I P} V_{A I P}
$$

The area averaged density and velocity at the AIP are given by $\rho_{A I P}$ and $V_{A I P}$ respectively. The baseline case in Fig. 14 has $A_{\infty} / A_{A I P}=0.725$ which shows that the stream tube ahead of the inlet is smaller than the inlet exit area indicating a deceleration of the flow ahead of inlet. For the high jet MFR case, the $A_{\infty} / A_{A I P}$ value of 0.719 indicates a slightly lower inlet mass flow rate than the baseline case.

Actuator configuration B is shown in Fig. 15 where the row of 14 jets are moved upstream near the entrance of the inlet at $x / L=0.13$. The high jet MFR case of $0.69 \%$ shows a much lower $D C 60$ value of 0.277 as compared to configuration A where $D C 60=0.367$. Figure 16 shows the area averaged $60^{\circ}$ sector total pressure ratio as the sector is rotated about the AIP for the baseline and jet configuration A

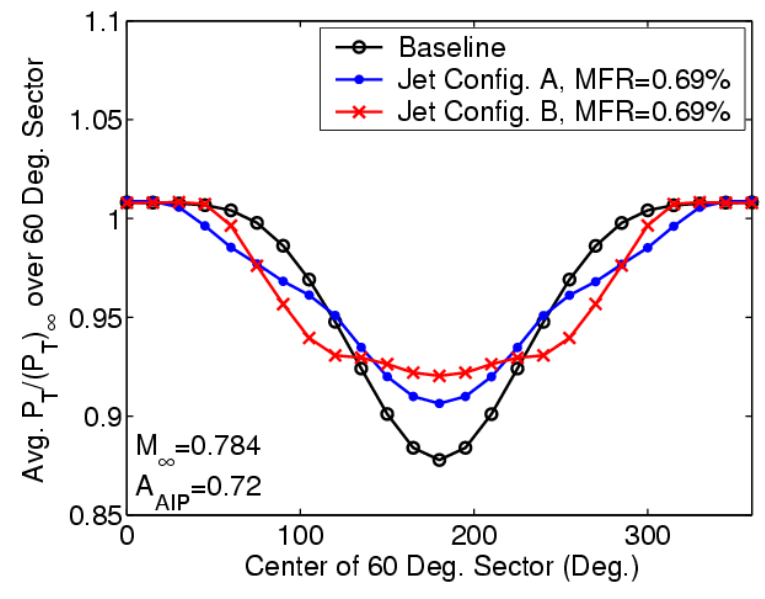

Figure 16. A comparison of the area averaged total pressure ratio for a $60^{\circ}$ sector rotated about the AIP between the baseline case and the controlled cases for jet configuration $A$ and $B$. and B cases. The 'worst' sector average total pressure, $\left(P_{\text {Tavg }}\right)_{\text {crit }}$, for all three cases is the minimum point at $180^{\circ}$ which is the bottom of the AIP. This comparison of the mean sector pressures shows how the jets in configuration B improve the distortion at the bottom of the inlet as compared to configuration A and the baseline. 


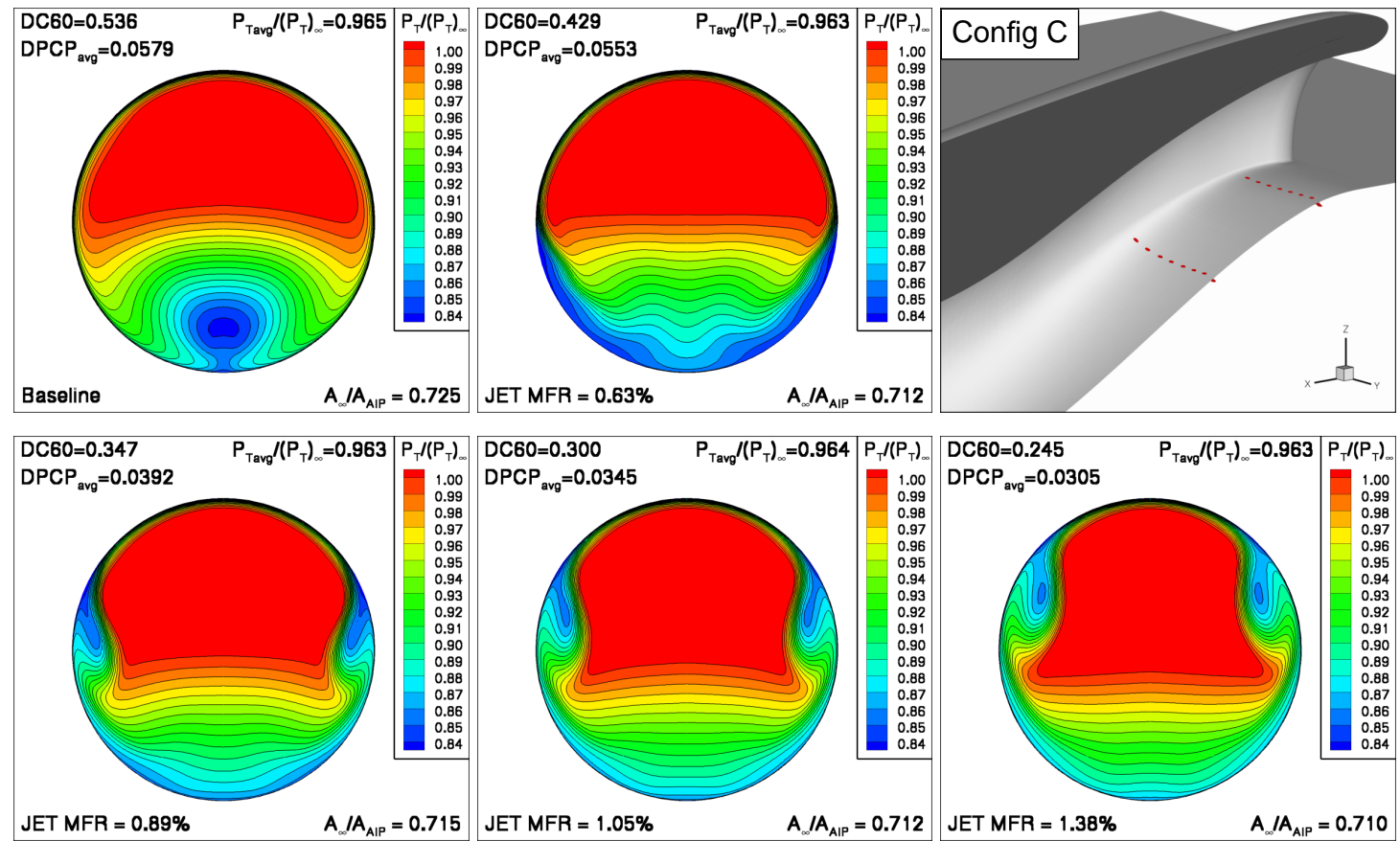

Figure 17. Total pressure ratio contours of the numerical simulations for the BLI inlet with 28 control jets for varying jet mass flow ratios where $M_{\infty}=0.784$ and $R e_{D}=13.8$ million. This case has two rows of jets on the bottom surface of the $B L I$ inlet at $x / L=0.13$ and $\mathbf{x} / \mathbf{L}=\mathbf{0 . 3 4}$.

A comparison of total pressure ratio contour plots for configuration A and B shows how the jet actuators placed closer to the entrance of the inlet improved the average pressure at the bottom of the inlet. However, configuration B is less effective in moving the low pressure along the sides of the inlet. It also shows how the $D P C P_{\text {avg }}$ only varied slightly (less than 5\%) where DC60 was reduced by about 30\% when moving the jets closer to the inlet entrance.

Configuration $\mathrm{C}$ is a combination of the two jet actuator locations, and has 28 jet actuators as shown in Fig. 17. The low jet MFR case is $0.63 \%$ with a $D C 60=0.429$ and a $D C P C_{a v g}=0.0553$. These distortion values are much higher than the two previous cases at the same jet MFR and with half the number of jets. This indicates that the momentum of the jets and not the total mass flow rate is the most important parameter for the effectiveness of the control jets. Increasing the jet MFR to $1.38 \%$ for this case results in $D C 60=0.245$ which is $15 \%$ lower than the single jet row case in Fig. 15 but at twice the jet MFR. The $D P C P_{\text {avg }}$ for the two jet row case at the high jet MFR in configuration $\mathrm{C}$ is $29 \%$ lower than high jet MFR for configuration B. This shows how improving one distortion descriptor does not necessarily result in an improvement of the other descriptor.

Figure 18 shows the location of the jets and numerical results for configuration $\mathrm{D}$ where 28 jets are located on the bottom and side of the inlet at $x / L=0.13$. The $1.37 \%$ jet MFR case had a $D C 60=0.255$ and a $D P C P_{\text {avg }}=0.0341$. Compared to jet configuration $\mathrm{C}$, this configuration has higher distortion values for the same amount of jet MFR. This shows that adding the jets on the bottom of the inlet downstream is more effective in reducing distortion than adding them on the side of the inlet upstream. Since the 'worst' $60^{\circ}$ sector is on the bottom of the inlet, it makes sense that adding jets on the side would be less effective than putting them on the bottom.

Configuration $\mathrm{E}$ is identical to $\mathrm{D}$ but with a downstream row of jets added on the duct bottom for a total of 42 jets, as shown in Fig. 19. The low jet MFR case of $1.34 \%$ had a $D C 60=0.334$ and a $D P C P_{\text {avg }}=0.0391$ which are much higher than configuration $\mathrm{C}$ for the same jet MFR. The high jet MFR for configuration $\mathrm{E}$ was $2.08 \%$ producing $D C 60=0.224$ which is only $9 \%$ lower than configuration $C$ at a jet MFR of $1.38 \%$. Configuration $\mathrm{E}$ for the MFR case of $1.34 \%$ had a $D P C P_{\text {avg }}=0.0391$ which is also only $9 \%$ lower than configuration $\mathrm{C}$ at $1.38 \%$ jet MFR. This comparison between configureations $\mathrm{C}$ and $\mathrm{D}$ shows how adding the side jets only results in a small improvement of the flow distortion while significantly increasing in the total jet MFR.

Jet configuration F shown in Fig. 20 has a more distributed pattern of jets on the bottom and side of the inlet. This case has 30 jets on the bottom and 10 jets on the side of the inlet near the corners. The total pressure contour plots 

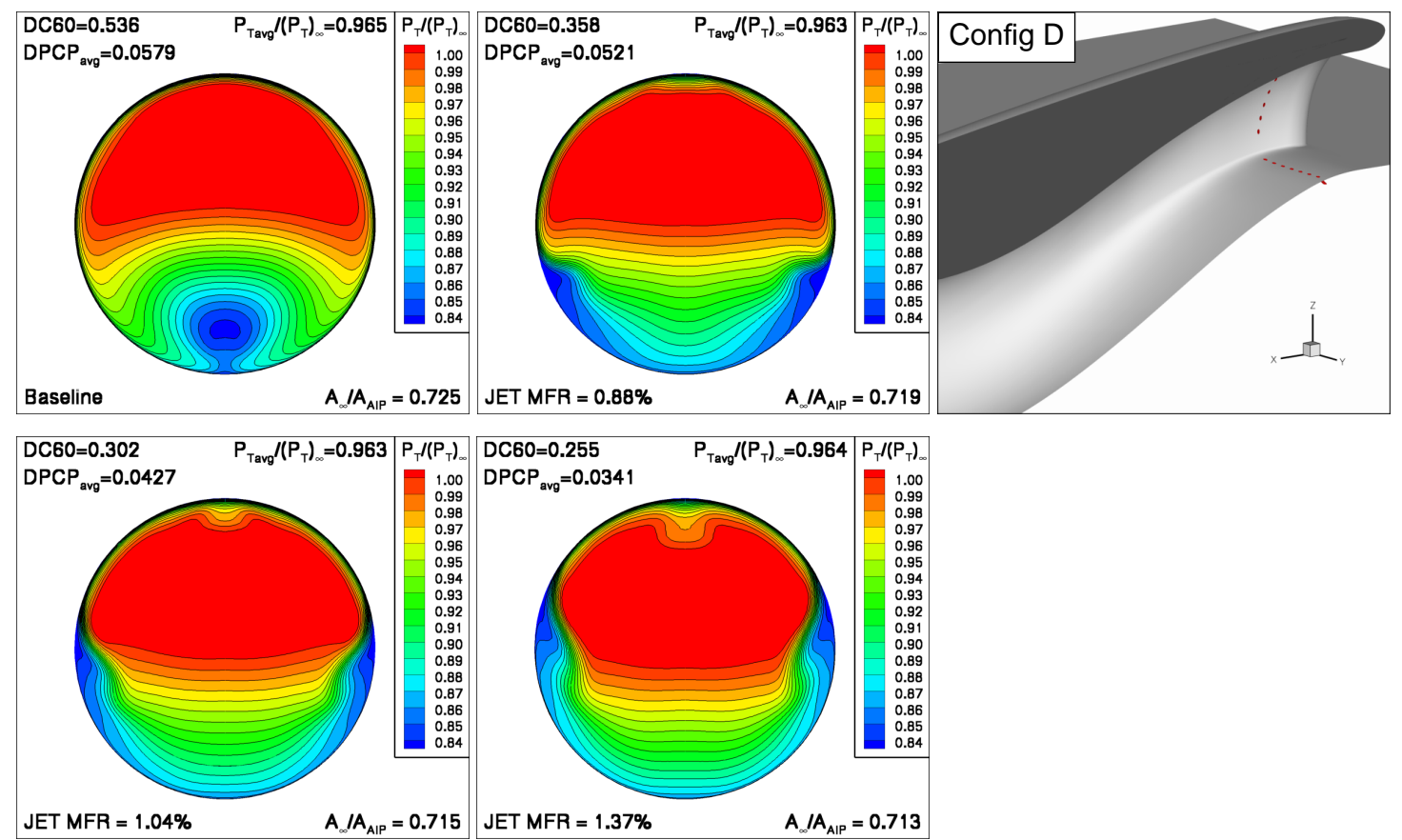

Figure 18. Total pressure ratio contours of the numerical simulations for the BLI inlet with 28 control jets for varying jet mass flow ratios where $M_{\infty}=0.784$ and $R_{D}=13.8$ million. This case has one row of jets near the entrance at $x / L=0.13$ with 14 jets on the bottom and 14 jets on the side of the BLI inlet.

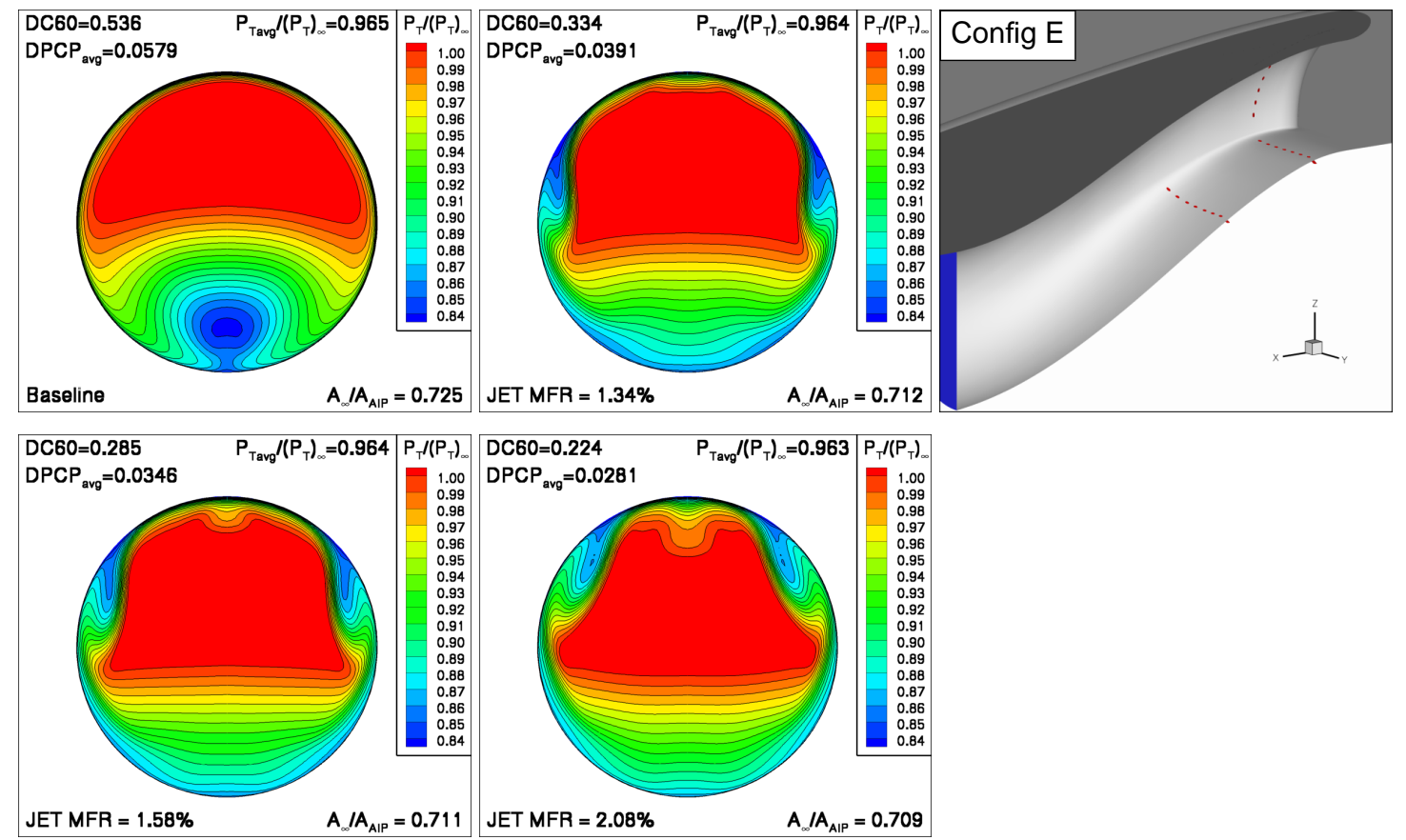

Figure 19. Total pressure ratio contours of the numerical simulations for the BLI inlet with 42 control jets for varying jet mass flow ratios where $M_{\infty}=0.784$ and $R_{D}=13.8$ million. This case has two rows of jets on the bottom surface of the $B L I$ inlet at $x / L=0.13$ and $x / L=0.34$ with a row of jets on the sides near the entrance at $x / L=0.13$. 

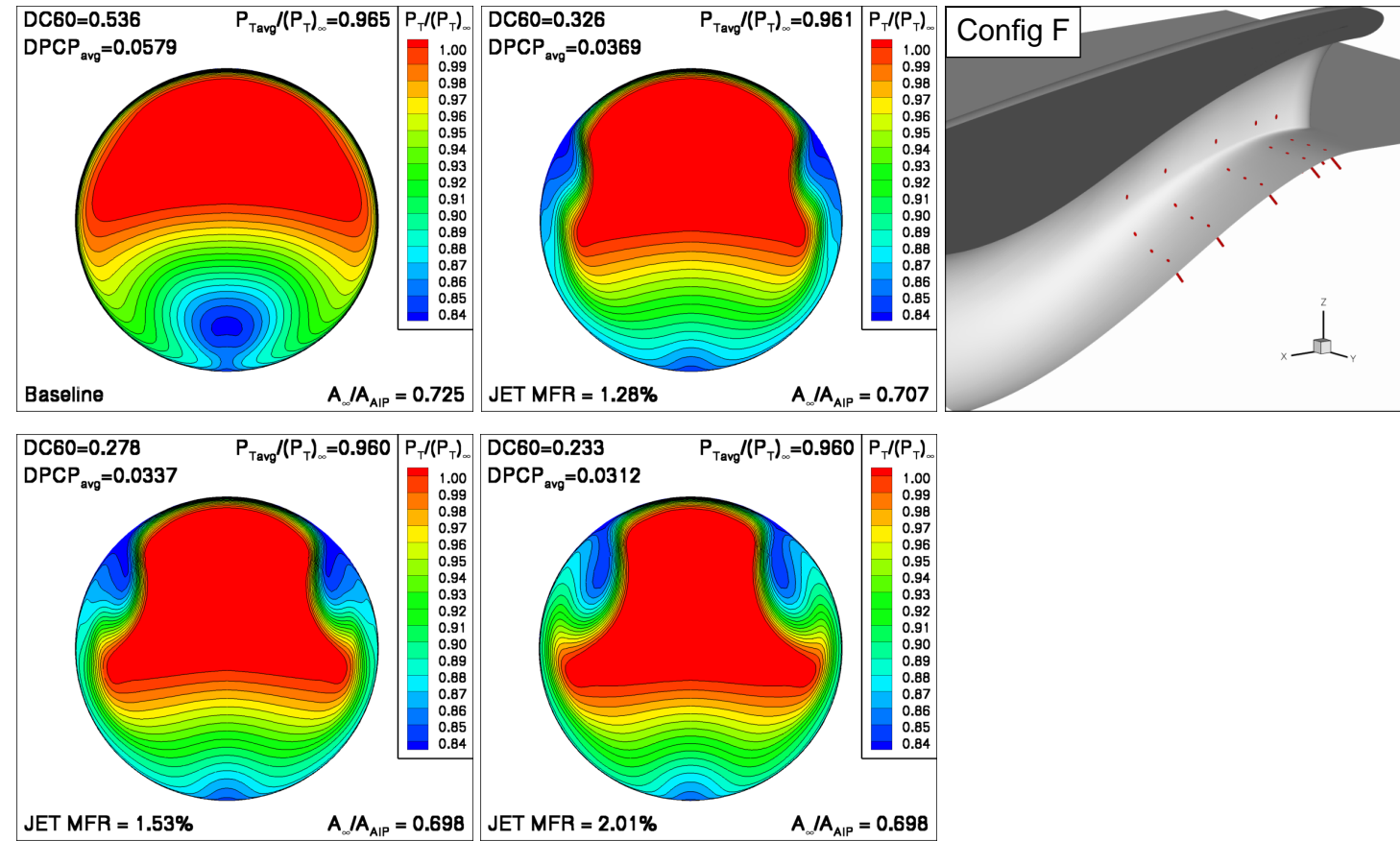

Figure 20. Total pressure ratio contours of the numerical simulations for the BLI inlet with 40 control jets for varying jet mass flow ratios where $M_{\infty}=0.784$ and $R_{D}=13.8$ million. This case has five rows of jets with each row having six jets on the bottom and two on the side of the BLI inlet.
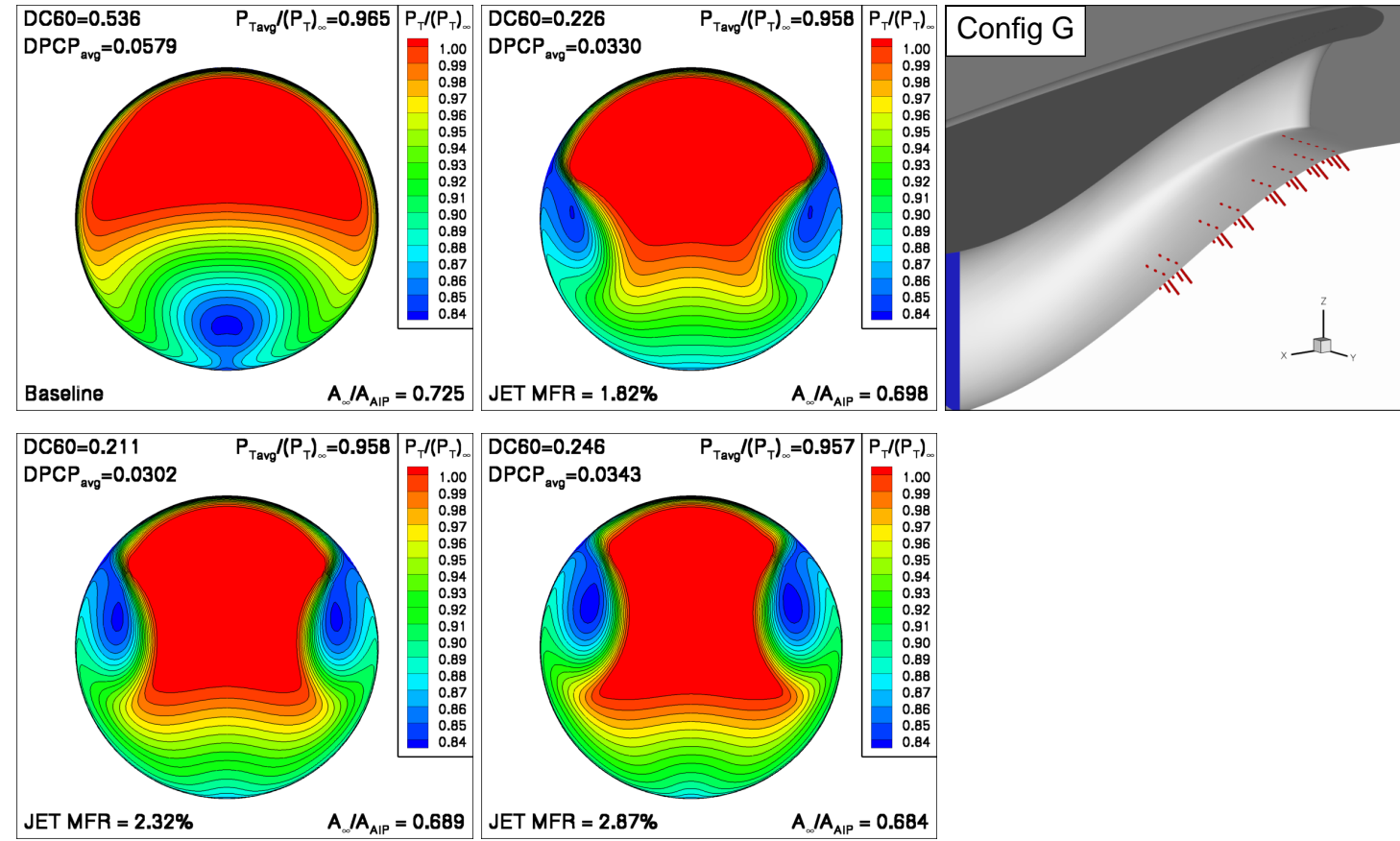

Figure 21. Total pressure ratio contours of the numerical simulations for the BLI inlet with 56 control jets for varying jet mass flow ratios where $M_{\infty}=0.784$ and $R_{D}=13.8$ million. This case has eight rows of jets on the bottom surface of the BLI inlet with the first row having 14 jets and the other rows with six jets concentrated at the center of the BLI inlet. 


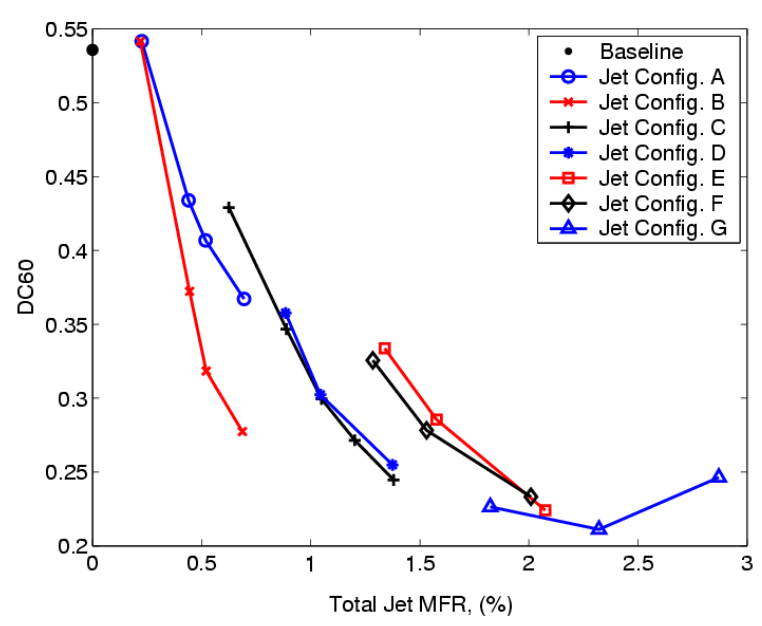

Figure 22. Summary of DC60 versus the total jet MFR for all of the jet configurations for the high Mach and Reynolds number cases.

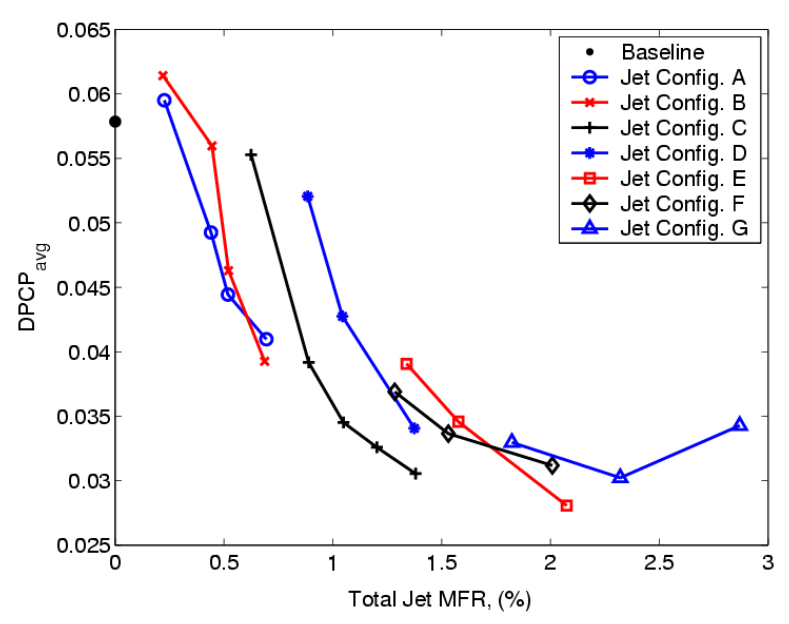

Figure 23. Summary of DPCP ${ }_{\text {avg }}$ versus the total jet MFR for all of the jet configurations for the high Mach and Reynolds number cases.

in Fig. 20 show that this actuator pattern produces two large low pressure regions on the side of the inlet AIP. The distortion for the high jet MFR case shows that DC60 is only slightly higher for the same jet MFR in configuration E. Likewise, $D P C P_{\text {avg }}$ is $14 \%$ higher when compared to the same jet MFR in configuration E. Jet configuration F turns out not to perform as well as configuration $\mathrm{E}$ because of the higher distortion values and the larger low pressure regions on the side of the inlet.

Configuration $\mathrm{G}$ looked at concentrating the jets at the bottom center of the inlet where the distortion is the highest. Figure 21 shows the location of the eight rows of jets for this configuration. This configuration had a total of 56 jets where the first row had 14 jets and the next seven rows had six jets concentrated near the bottom centerline of the inlet. The low jet MFR case was $1.82 \%$ and had a DC60 distortion of 0.226 and a $D P C P_{a v g}$ of 0.0330 . The jets on the centerline do a good job of clearing out the center but create large low pressure regions on the side of the inlet. These regions produce the worst $60^{\circ}$ sector. Increasing the jet MFR to $2.32 \%$ improves the distortion at the bottom of the AIP and moves the low pressure regions higher along the sides. The $60^{\circ}$ average sector pressure for these low pressure regions improves slightly lowering $D C 60$ to 0.211 . The $2.32 \%$ jet MFR case also reduces the $D P C P_{a v g}$ to 0.0302 . Increasing the jet MFR to $2.87 \%$ increases the size of the low pressure regions on the side of the inlet without changing the flow on the bottom of the AIP. This increase in the size of the low pressure regions increases the distortion values where $D C 60=0.246 \%$ and $D P C P_{\text {avg }}=0.0343$. All three controlled cases caused a small reduction in the pressure recovery compared to the baseline case.

A plot of the distortion descriptors versus the total jet MFR for each of the jet configurations is shown in Fig. 22 and 23. The comparison of the DC60 distortion in Fig. 22 shows how none of the configurations were able to reduce DC60 below 0.2. Comparing configuration A with B indicates that moving the row of jets from $x / L=0.34$ to 0.13 greatly improved DC60 for the same number of jets at the same jet MFR. The other configurations indicate, that by adding more jets and increasing the total jet MFR, predicted DC60 can be decreased another 0.065 but at the cost of tripling the total jet MFR. The lowest predicted DC60 was 0.211 for jet configuration G with a total jet MFR of $2.32 \%$. The best jet configuration for a jet MFR under $1 \%$ was configuration $\mathrm{B}$ where $D C 60=0.277$ for a jet $\mathrm{MFR}=0.69 \%$.

Figure 23 shows the trend of $D P C P_{a v g}$ versus jet MFR for the controlled high Mach number cases. This plot shows that $D P C P_{\text {avg }}$ bottoms out at 0.0281 for a jet MFR of $2 \%$ using configuration $\mathrm{E}$. This figure also indicates that there is little difference between $D P C P_{a v g}$ for configurations $\mathrm{A}$ and $\mathrm{B}$ and how the combination of the two, in configuration $\mathrm{C}$, continues the decreasing trend of $D P C P_{a v g}$ for increasing jet MFR. Figure 23 also shows how the low jet MFR cases for configuration $\mathrm{C}$ and $\mathrm{D}$ did not perform as well as $\mathrm{A}$ and $\mathrm{B}$ for a given jet MFR.

A summary of the distortion descriptors in terms of total jet momentum coefficient, $C_{\dot{m} \text { jet }}$, are shown in Figs. 24 and 25 , where the total jet momentum coefficient is defined in (6). 


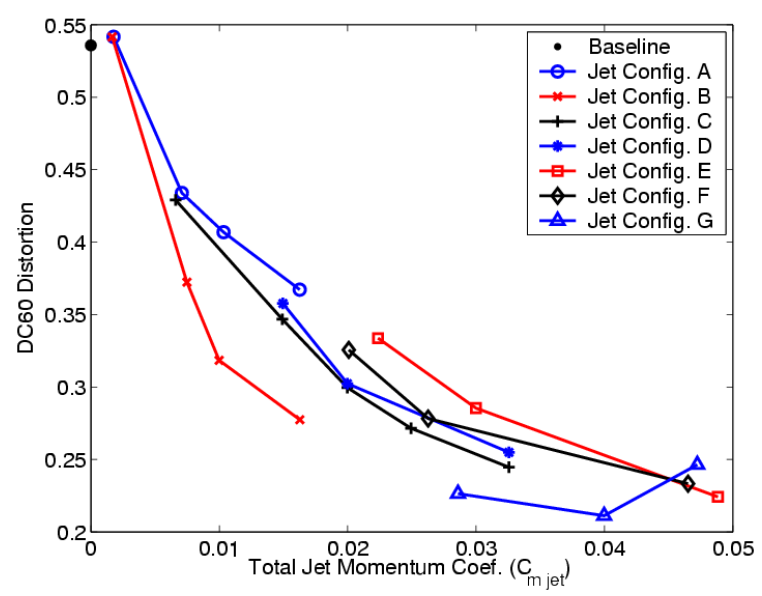

Figure 24. Summary of DC60 versus the total jet momentum coefficient, $C_{\text {mjet }}$, for all of the jet configurations for the high Mach and Reynolds number cases.

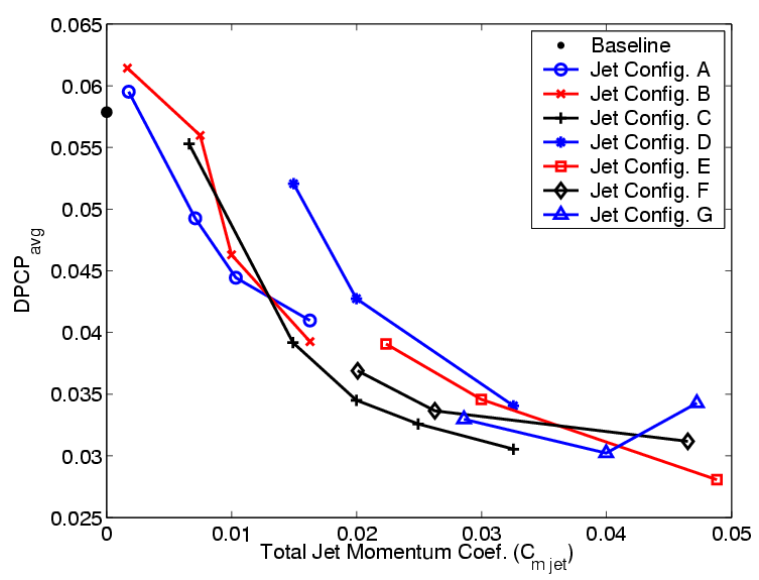

Figure 25. Summary of DPCP ${ }_{\text {avg }}$ versus the total jet momentum coefficient, $\mathbf{C}_{\text {mjet }}$, for all of the jet configurations for the high Mach and Reynolds number cases.

$$
C_{\dot{m} j e t}=\frac{\text { Total Jet Momentum }}{\rho_{\infty} U_{\infty}^{2} A_{A I P}}
$$

These figures indicate that distortion is more a function of the total jet momentum as opposed to the jet mass flow ratio. As shown in Fig. 24, all but two configurations tend to fall on the same curve. Configurations B and G have lower distortion values for the same jet momentum as compared to the other configurations. Figure 25 plots $D P C P_{\text {avg }}$ as a function of the total jet momentum and all of the cases tend to fall on the same curve. Only the low jet MFR case for configuration D seems to fall off of this curve with higher $D P C P_{\text {avg }}$ values. These figures indicate a trend where the distortion starts to flatten out for increasing total jet momentum.

\section{Summary}

The objective of this research was to evaluate the ability of a RANS flow solver to predict the flow quality at the location of the engine face for a BLI offset inlet for baseline and flow controlled cases. Once the numerical approach was validated, the performance of VG jets, for the BLI inlet at high Mach and Reynolds numbers were evaluated. These numerical simulations will then provide guidance on the design of a future wind tunnel test. The second objective of the study was to provide insight into the flow physics of the flow control actuators for the development of a source term actuator model.

The BLI offset inlet geometry was generic in nature so that it would be an open geometry and still be representative of an inlet for a commercial version of the BWB aircraft. Numerical simulations were compared to two different experimental tests using this inlet geometry. The experiments were setup such that the inlet was ingesting a boundary layer with a height of at least $30 \%$ of the inlet height. The experimental data consisted of a high Mach and Reynolds number test for a baseline inlet (i.e. no flow control devices) performed in NASA Langley's 0.3-Meter Transonic Cryogenic Tunnel. A low speed test in NASA Langley's Basic Aerodynamics Research Tunnel (BART) provided data for the BLI inlet with passive and active flow control devices.

Overall, the numerical results compared well with the experiments for both the baseline and controlled cases. The baseline flow distortion for the high Mach and Reynolds cases were over predicted by the flow solver. This was related to the flow solver predicting a slightly lower minimum total pressure than was measured in the experiment. Despite this difference in the distortion, the flow solver was able to predict the pressure recovery well. Overall, the flow features in the contour plots of the total pressure compared well with experiments.

The low Mach and Reynolds number comparisons showed that the flow solver under predicted the baseline flow distortion by $13 \%$. However, the contour plots showed very similar flow features and compared very well with the experiments. A comparison of the duct flow using passive flow control devices, VG vanes, showed good agreement 
between the CFD and the low Mach number experimental data. Simulations of the VG jets were compared to an actuator mass flow sweep performed in the low Mach number experiment. This comparison indicated that the flow solver was able to predict the inlet distortion for varying actuator mass flow. The flow solver was also able to predict the actuator mass flow rate at the minimum distortion point.

The performance of the VG jets for the BLI inlet at high Mach and high Reynolds numbers were evaluated for several different actuator locations. These simulations indicated that a minimum DC60 of 0.211 could be achieved at a cost of $2.32 \%$ of the inlet mass flow. The simulations also predicted a minimum $D P C P_{\text {avg }}$ of 0.0281 using $2.08 \%$ of the inlet mass flow. From an inlet design point of view, a DC60 under 0.10 is desirable while using less than $1 \%$ of the inlet mass flow. The simulations for the high Mach number case indicate that more research needs to be performed on the location of the actuators in order to reduce the flow distortion further. Also the simulations showed that an inlet mass flow of $2 \%$ or more may be needed to acieve a DC60 value less than 0.10

The cost of fully griding the jet actuators was very high so only a few actuator patterns could be explored in this study. The results from these simulations will be used to validate a source term modeling approach which will greatly reduce the cost of modeling the jet actuators for the BLI inlet. ${ }^{6}$

\section{Acknolwegements}

This research was supported by the NASA Ultra Efficient Engine Technology (UEET) Highly Integrated Inlet (HII) project. Special thanks to Ms. Susan Gorton and Mr. Scott Anders for their work and discussions on the experimental tests and data. Also a special thanks to Dr. Pieter Buning for our discussion on the numerical flow solutions.

\section{References}

\footnotetext{
${ }^{1}$ Brown, S. A., "HSR Work Propels UEET Program (High Speed Research in Ultraefficient Engine Technology in Aircraft Industry)," Aerospace America, Vol. 37, No. 5, 1999, pp. 48-50.

${ }^{2}$ Liebeck, R. H., "Design of the Blended-Wing-Body,” AIAA Paper 02-0002, January 2002.

${ }^{3}$ Berrier, B. L. and Allan, B. G., "Experimental and Computational Evaluation of Flush-Mounted, S-Duct Inlets," AIAA Paper 04-0764, January 2004.

${ }^{4}$ Gorton, S. A., Owens, L. R., Jenkins, L. N., Allan, B. G., and Schuster, E. P., “Active Flow Control on a Boundary-Layer-Ingesting Inlet," AIAA Paper 04-1203, January 2004.

${ }^{5}$ Anabtawi, A. J., Blackwelder, R. F., Lissaman, P. B. S., and Liebeck, R. H., "An Experimental Investigation of Boundary Layer Ingestion in a Diffusing S-Duct With and Without Passive FLow Control," AIAA Paper 99-0739, January 2002.

${ }^{6}$ Waith, K. A., "Source Term Model for Vortex Generator Vanes in a Navier-Stokes Computer Code," AIAA Paper 2004-1236, January 2004.

${ }^{7}$ Buning, P. G., Jespersen, D. C., Pulliam, T. H., Klopfer, W. M., Chan, W. M., Slotnick, J. P., Krist, S. E., and Renze, K. J., "OVERFLOW User's Manual Version 1.8m," Tech. rep., NASA Langley Research Center, 1999.

${ }^{8}$ Jespersen, D. C., Pulliam, T. H., and Buning, P. G., "Vortex Generator Modeling for Navier-Stokes Codes," AIAA 97-0644, 1997.

${ }^{9}$ Pulliam, T. H. and Chaussee, D. S., "A Diagonal Form of an Implicit Approximate-Factorization Algorithm," Journal of Computational Physics, Vol. 39, February 1981, pp. 347-363.

${ }^{10}$ Steger, J. L., Dougherty, F. C., and Benek, J. A., "A Chimera Grid Scheme," Advances in Grid Generation, edited by K. N. Ghia and U. Ghia, Vol. 5 of FED, ASME, New York, NY, 1983.

${ }^{11}$ Menter, F., “Improved Two-Equation Turbulence Models for Aerodynamic Flows,” Tech. Rep. TM 103975, NASA, NASA Langley Research Center, Hampton, VA 23681-2199, 1992.

${ }^{12}$ Allan, B. G., Yao, C. S., and Lin, J. C., "Numerical Simulations of Vortex Generator Vanes and Jets on a Flat Plate," AIAA Paper 02-3160, June 2002.

${ }^{13}$ Murphy, K., Buning, P., Pamadi, B., Scallion, W., and Jones, K., "Status of Stage Separation Tool Development for Next Generation Launch Technologies," AIAA paper 04-2595, June 2004.

${ }^{14}$ Chan, W. M. and Gomez, R. J., "Advances in Automatic Overset Grid Generation Around Surface Discontinuities," AIAA Paper 99-3303, July 1999.

${ }^{15}$ Seddon, J. and Goldsmith, E. L., Intake Aerodynamics, second Edition, AIAA, Reston, Virginia, 1999.
} 